\title{
Integrins mediate placental extracellular vesicle trafficking to lung and liver in vivo
}

\author{
Sean L. Nguyen ${ }^{1,2}$, Soo Hyun Ahn ${ }^{3}$, Jacob W. Greenberg ${ }^{4}$, Benjamin W. Collaer ${ }^{5}$, \\ Dalen W. Agnew ${ }^{3}$, Ripla Arora ${ }^{6}$ \& Margaret G. Petroff ${ }^{1,3,4}$
}

Membrane-bound extracellular vesicles (EVs) mediate intercellular communication in all organisms, and those produced by placental mammals have become increasingly recognized as significant mediators of fetal-maternal communication. Here, we aimed to identify maternal cells targeted by placental EVs and elucidate the mechanisms by which they traffic to these cells. Exogenously administered pregnancy-associated EVs traffic specifically to the lung; further, placental EVs associate with lung interstitial macrophages and liver Kupffer cells in an integrin-dependent manner. Localization of EV to maternal lungs was confirmed in unmanipulated pregnancy using a transgenic reporter mouse model, which also provided in situ and in vitro evidence that fetally-derived EVs, rarely, may cause genetic alteration of maternal cells. These results provide for the first time direct in vivo evidence that placental EVs target maternal immune cells, and further, that EVs can alter cellular phenotype.

Extracellular vesicles (EVs), which can be secreted as exosomes, microvesicles, and apoptotic bodies, are increasingly recognized as a mechanism of intercellular communication in all organisms ${ }^{1}$. Each type of EV possesses unique cargo and biological activity, which establishes a need for acquiring a basic understanding of their role in physiologic and pathologic processes. Exosomes are 40-150 nm membrane-enclosed EVs created by the invagination of early endosomes to form multivesicular bodies, which then can fuse with either the lysosome or the plasma membrane. When they fuse with the plasma membrane, the intraluminal vesicles are released from the cell as exosomes, carrying bioactive nucleic acids, proteins, and lipids through interstitial space and the circulation to distant cells ${ }^{2}$. Both the cell of origin and their physiological state strongly influence exosome quantities and activity.

Among mammalian cells and tissues that shed vesicles, the placenta plays a particularly important role, releasing enormous quantities of EVs in order to communicate with local and distant maternal cells during pregnancy. This is particularly true of hemochorial species, in which the maternal-fetal interface consists of trophoblast cells that contact maternal blood. Humans and mice both possess this type of placentation, with the chorionic villi and labyrinth, respectively, possessing outermost syncytiotrophoblast cells that release EVs into the maternal circulation ${ }^{3,4}$. Because of this intimate association, placental EVs have the potential to communicate with any tissue in the mother that they are able to access. As gestation advances, placental exosome concentration increases in maternal plasma ${ }^{5,6}$; additionally, their bioactivity may change in pathological conditions including preeclampsia and gestational diabetes ${ }^{7-9}$. These changes have spurred interest into possible roles of exosomes in regulating immune and cardiovascular responses to pregnancy and parturition. Further, interest in placental EVs as biomarkers for these and other complications has burgeoned ${ }^{10}$.

In vitro studies have suggested that placental exosomes can be internalized by and affect activity of multiple immune cell types. Placental exosomes can induce migration and cytokine secretion by macrophages ${ }^{11}$, suppress NK cells and T cells ${ }^{12-14}$, protect cells against viral infection ${ }^{15}$, and may promote spiral artery remodeling ${ }^{16}$. However, only a few studies have queried their trafficking patterns and functional effects in vivo. Tong et al. ${ }^{17}$

${ }^{1}$ Cell and Molecular Biology Program, College of Natural Science, Michigan State University, 3009 Interdisciplinary Science and Technology Building 766 Service Road, East Lansing, Ml 48854, USA. ${ }^{2}$ Institute for Integrative Toxicology, Michigan State University, East Lansing, MI, USA. ${ }^{3}$ Department of Pathobiology and Diagnostic Investigation, College of Veterinary Medicine, Michigan State University, East Lansing, MI, USA. ${ }^{4}$ Department of Microbiology and Molecular Genetics, Michigan State University, East Lansing, MI, USA. ${ }^{5}$ Department of Biochemistry and Molecular Biology, Michigan State University, East Lansing, MI, USA. 'Department of Obstetrics, Gynecology, and Reproductive Biology, Institute for Quantitative Health Science and Engineering, Michigan State University, East Lansing, MI, USA.『email: Petrof10@msu.edu 
found localization of human placental exosomes in the lungs, kidney, and liver of mice $24 \mathrm{~h}$ after intravenous injection. On the other hand, intraperitoneally injected exosomes purified from plasma of pregnant mice traffic to the uterus and cervix, and further, can cross into the fetus ${ }^{18}$. In this study, we advance our understanding of placental EVs by identifying specific cellular targets of EVs and the molecular mechanisms that influence their trafficking. We also developed a Cre-recombinase reporter system in unmanipulated pregnancy to support these observations. Finally, we provide in vivo and in vitro proof of principle that genetic alteration of maternal cells by placenta-derived exosomes is possible.

\section{Results}

Pregnant plasma localizes to lung in vivo. Consistent with our prior results ${ }^{6}$, the concentration of EVs in the plasma of gestation day (GD) 14.5 pregnant mice were $~ 2.4$-fold higher than in nonpregnant females $(P=0.015)$ (Suppl. Figure 1). As placental EVs are a likely source of this increase, and as they can potentially access all maternal organs via the vasculature, we tested the specificity of their trafficking by comparing localization of plasma EVs from nonpregnant and pregnant mice. To this end, EVs were purified from plasma, labeled with the red fluorescent dye PKH26, and administered into the tail vein of nonpregnant recipient females (Fig. 1a). The lung and liver of recipients were harvested after $30 \mathrm{~min}$ and evaluated by epifluorescence microscopy. In the lung, only EVs from pregnant mice were readily detectable; EVs from nonpregnant mice were scarce or undetectable (Fig. 1b,c). The same trend did not hold true for EV localization to the liver (Fig. 1d,e). These results suggest preferential localization of EVs associated with pregnancy to the lung.

Placental EVs traffic to lung interstitial macrophages in vivo. We next sought to identify the type(s) of cells in the lung with which placental EVs associate. To this end, we developed an explant system in which placental EVs are purified following $18 \mathrm{~h}$ of culture at ambient oxygen concentration (Suppl. Figure 2). EVs from the supernatant of GD14.5 placental explants were isolated, labeled with PKH26, and administered intravenously into nonpregnant female mice (Fig. 2a). After $30 \mathrm{~min}$, lungs were harvested and dispersed for flow cytometry. For unbiased identification of immune cells in the lung that associated with PHK26, we used $\mathrm{t}$-distributed stochastic neighbor embedding ( $\mathrm{t}$-SNE). This approach identified CD45+ cells, and within these, cells positive for PKH26 (Fig. 2b). PKH26-positive cells were also CD45+, MHCII ${ }^{\text {hi/lo }}$, F4/80+, autofluorescence (AF)-, CD11 $c^{\mathrm{lo}}$, and CD64+-all characteristic of interstitial macrophages (Fig. 2b) ${ }^{19}$. We confirmed this result first by immunofluorescence microscopy, in which PKH26 colocalized with LYVE1+ and CD68+ interstitial macrophages (Fig. 2c) ${ }^{19}$. Second, using forward-gating strategy to complement t-SNE analysis ${ }^{20}$, we found that PKH26 + placental EVs localize predominantly with interstitial, but not alveolar, macrophages (Fig. 3). Confocal microscopy of liver tissue from the same animals revealed localization of placental EVs within CD31+ endothelial cells and F4/80+ Kupffer cells (Suppl. Figure 3A, 3B).

Outer membrane proteins including integrins influence trafficking of placental EVs to the lung and liver. We next asked whether outer membrane components, specifically integrins, influence trafficking of placental EVs, since these proteins can mediate the trafficking of tumor-derived EVs to specific organs ${ }^{21}$. A survey of multiple integrins revealed that placental EVs express ITG $\alpha 3, \alpha \mathrm{V}, \alpha 5, \beta 1$, and $\beta 3$, whereas ITG $\beta 6$ was absent from EVs despite its expression in the placenta (Fig. 4a). As expected, proteinase K treatment of EVs abolished immunoreactivity for all membrane-associated integrins examined, although neither expression of the exosome marker CD9 nor exosome morphology were affected (Fig. 4a,b).

To determine whether $\mathrm{pEV}$ localization to lung and liver was mediated by outer membrane proteins, EVs were digested with proteinase $\mathrm{K}$, labeled, and administered into nonpregnant mice. Proteinase $\mathrm{K}$ abolished localization of EVs to the lung and liver (Fig. 4c-f) of EV-treated mice. To account for the possibility that proteinase K caused degradation of EVs, we also administered tenfold more EVs, and still observed vastly reduced numbers of EVs in liver and lung (Fig. 4d,f). Consistent with this observation, treatment with proteinase $\mathrm{K}$ to remove outer membrane proteins reduced the localization of EVs to lung interstitial macrophages (Suppl. Figure 4).

To test the hypothesis that integrins mediate placental EV targeting to the lung, we pre-incubated EVs with RGD and HYD-1 peptides, which block binding of integrins $\alpha 5 \beta 1 / \alpha \mathrm{V} \beta 3$ and $\alpha 3 \beta 1$ respectively, to their substrates ${ }^{22,23}$ prior to intravenous administration into nonpregnant recipients. Mice were sacrificed $30 \mathrm{~min}$ after treatment, and lung and liver were analyzed by fluorescence microscopy (Fig. 4c, e). Although proteinase $\mathrm{K}$ ablated localization of placental EVs to both lung (Fig. 4c, upper right panel) and liver (Fig. 4e, upper right panel), placental EVs remained detectable in both tissues after pretreatment with integrin-blocking peptides (Fig. 4c,e, lower left panels). In the lung, neither peptide significantly reduced the total numbers of placental EVs (Fig. 4d). In the liver, however, RGD pretreatment resulted in a significant reduction of placental EV localization (Fig. 4f).

Although the total numbers of placental EVs that localized to the lung were not reduced by HYD-1, we noticed that the tissue distribution of the vesicles was altered, with EVs appearing to remain within large vessels (Fig. 4c, lower left panel). To better characterize the effect of HYD-1 treatment on placental EV localization to the lung, 200-micron lung sections were cleared by CUBIC and imaged by $3 \mathrm{D}$ confocal microscopy ${ }^{24}$. Strikingly, we saw localization of placental EVs exclusively within the vasculature, suggesting that they were blocked from entering the interstitial tissue (Fig. 4g,h).

Since HYD-1 pretreatment inhibited rapid migration of placental EVs to lung interstitium, we asked whether this inhibition is sustained. Using Li-Cor whole organ imaging as a high throughput screening method, we labeled EVs with near-infrared (NiR) dye, administered them i.v. into nonpregnant recipients, and quantified vesicle localization (Fig. 5a). Untreated EVs remained detectable in the lung after $24 \mathrm{~h}$, but pretreatment with HYD-1 significantly decreased their presence (Fig. 5b). RGD pretreatment tended to reduce localization to the lung at $24 \mathrm{~h}$, but the result was not statistically significant. 

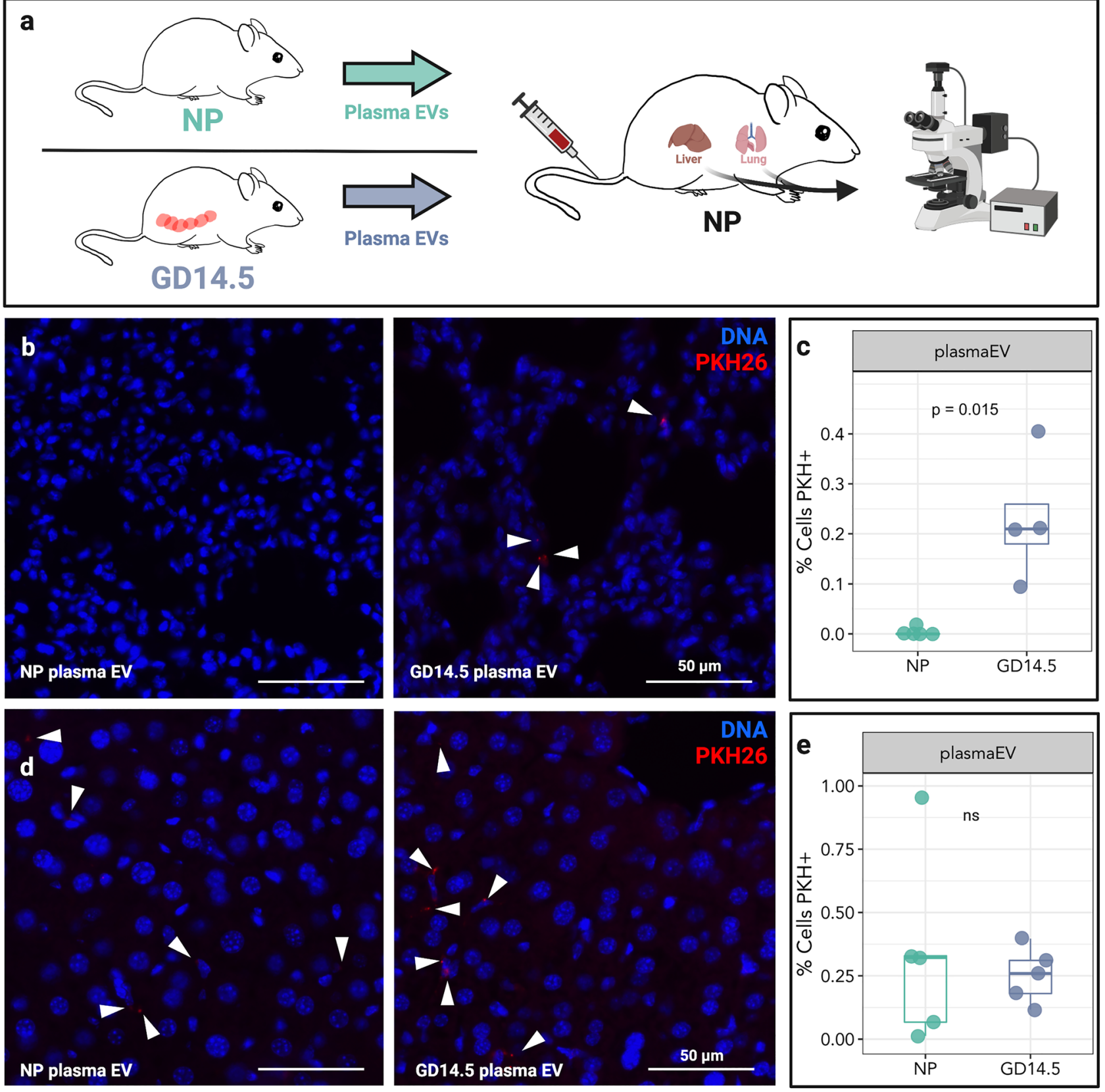

Figure 1. Pregnant plasma EVs traffic to murine lungs and liver in vivo. (a) Plasma EVs $\left(2.5 \times 10^{10}\right)$ isolated from the plasma of nonpregnant (NP) or GD14.5 pregnant mice were labeled with PKH26 and administered i.v. into NP mice. Mice were sacrificed after $30 \mathrm{~min}$, and lung and liver were analyzed by epifluorescence microscopy. (b) Fluorescence microscopy of lung from a mouse treated with plasma EVs from nonpregnant (NP, left; $\mathrm{n}=5$ ) or GD14.5 pregnant (right; $\mathrm{n}=4$ ) mice (Arrowheads, EVs). Images are representative of five random fields examined in each animal. (c) Quantification of plasma EVs as evidenced by presence of red fluorescence in recipient lung. Dots represent biological replicates, Wilcoxon test. (d) Fluorescence microscopy of liver from a mouse treated with plasma EVs from nonpregnant (NP, left; $n=5$ ) or GD14.5 pregnant (right, $n=5)$ mice EVs (Arrowheads, plasma EVs). Images are representative of five random fields examined in each animal. (e) Quantification of plasma EVs as evidenced by presence of red fluorescence in recipient liver. Dots represent biological replicates, Wilcoxon test.

We also imaged other organs including, the brain, thymus, heart, kidney, paraaortic lymph node, spleen, small intestine, uterus, and ovaries, again reasoning that EVs in the maternal vasculature can potentially access any maternal organ. Twenty-four hours after administration of NIR-labeled placental EVs, we did not observe any significant changes in NIR signal relative to the vehicle control treatments (Fig. 5c). This result suggests that placental EVs are not detectable in these tissues using this model. 

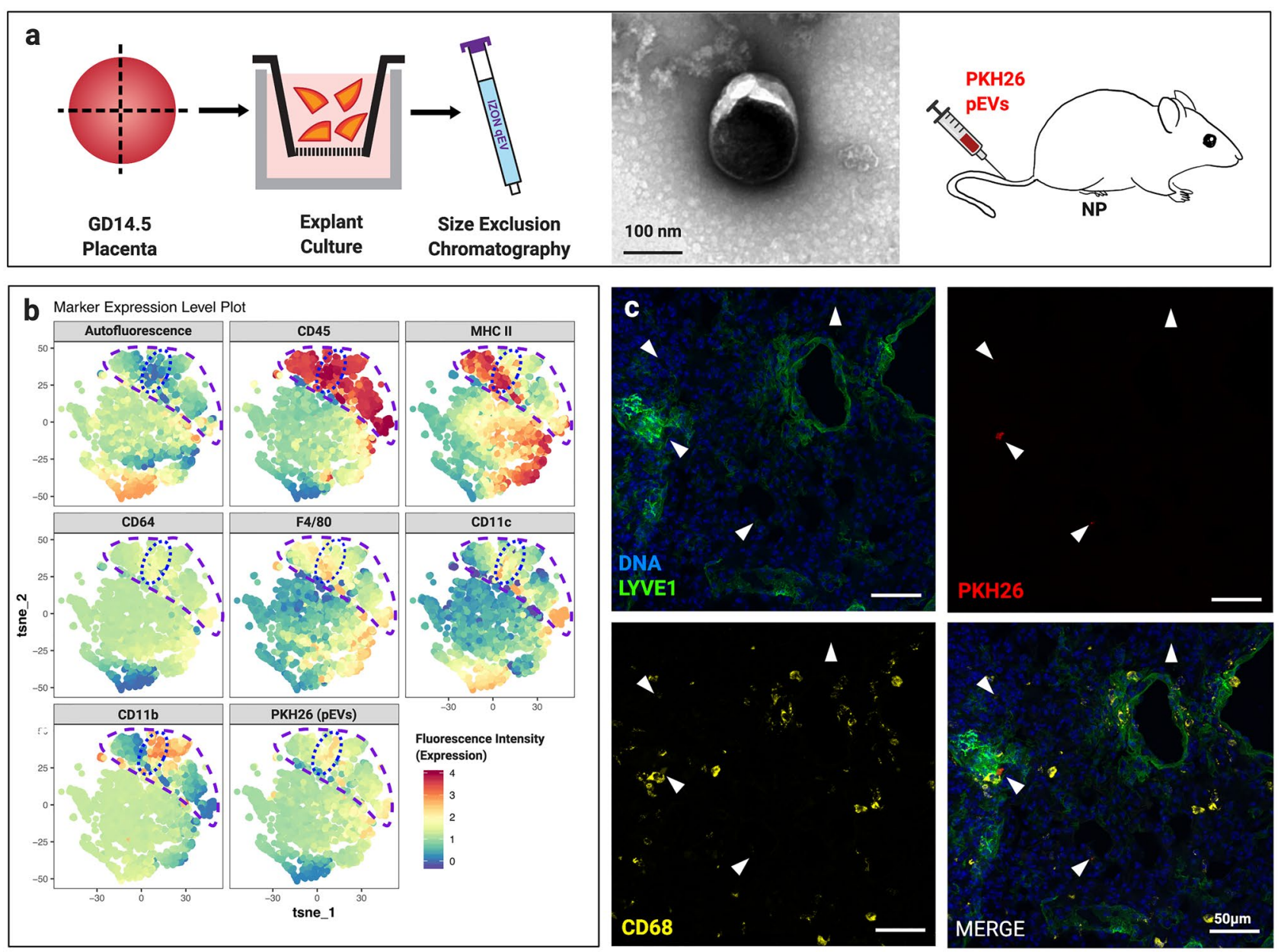

Figure 2. Placental EV trafficking in vivo. (a) EVs were purified from the medium of GD14.5 placental explants; a representative TEM is shown. After labeling with PKH26, $2.5 \times 10^{10} \mathrm{EVs}$ were administered i.v. into nonpregnant females. After $30 \mathrm{~min}$ mice were sacrificed, and lung and liver harvested and processed for downstream analysis. (b) t-SNE analysis of dispersed and murine lung cells after treatment of mice with GD14.5 placental EVs. Cell suspensions were stained with the fluorophore-conjugated antibodies indicated above each panel and analyzed by flow cytometry. Experiments shown is representative of seven independent experiments. (c) Immunofluorescence microscopy of lungs from mice treated with GD14.5 placental EVs. Arrows, pEV foci (red), one of which in this image colocalizes with LYVE1 (green) and CD68 (yellow). Representative images of five random fields per animal; $\mathrm{n}=7$ in three independent experiments.

Placental EVs localize to maternal lung in unmanipulated pregnancy and may genetically modify maternal lung cells. The above studies support the notion that placental EVs traffic to the maternal lung. However, a single bolus of purified EVs does not recapitulate the continual release of EVs from the placenta nor their sustained presence in maternal circulation. To address this limitation, we generated a model in which fetal EVs could be detected within the context of normal pregnancy. $\mathrm{mTmG}$ mice ${ }^{25}$ express a fluorescent reporter construct that, in the absence of Cre recombinase, allows constitutive expression of membrane-targeted red fluorescent protein, tandem dimer Tomato (mTomato). In the presence of Cre, the mTomato locus is excised, resulting in the expression of membrane-targeted enhanced green fluorescent protein (EGFP; mGFP). By mating $m$ TmG females with CMV-Cre males (Fig. 6a), female fetuses inherit both the CMV-Cre and mTmG loci, and thus express mGFP in all tissues, including the placenta. Male embryos inherit the wild type (WT) locus and continue to express the mTomato red fluorescent protein. Confocal microscopy (Fig. 6b) and western blot analysis (Fig. 6c) confirmed expression of mGFP in both placentas and placental EVs from female CMV-Cre;mTmG but not WT;mTmG fetuses. Both CMV-Cre;mT/mG and WT;mT/mG placentas and $\mathrm{pEV}$ samples expressed the control protein, TSG101.

Using this model, we asked whether fetal/placental mGFP + EVs are carried to the lung. Using confocal microscopy, we readily detected mGFP fluorescence in maternal lung from GD14.5 CMV-Cre-mated mTmG females (Fig. 6d). Most of the signal we observed was punctate and associated with maternal mTomato-expressing cells, similar to what was observed when labeled placental EVs were administered exogenously. Interestingly, we also observed mGFP expression that was clearly membrane-associated, surrounding distinct DAPI-stained nuclei (Fig. 6e,f). These cells did not express mTomato, indicating that they had undergone Cre-mediated recombination. We observed mGFP-associated foci and cells only in $\mathrm{mT} / \mathrm{mG}$ dams mated to CMV-Cre males, and not in 
a
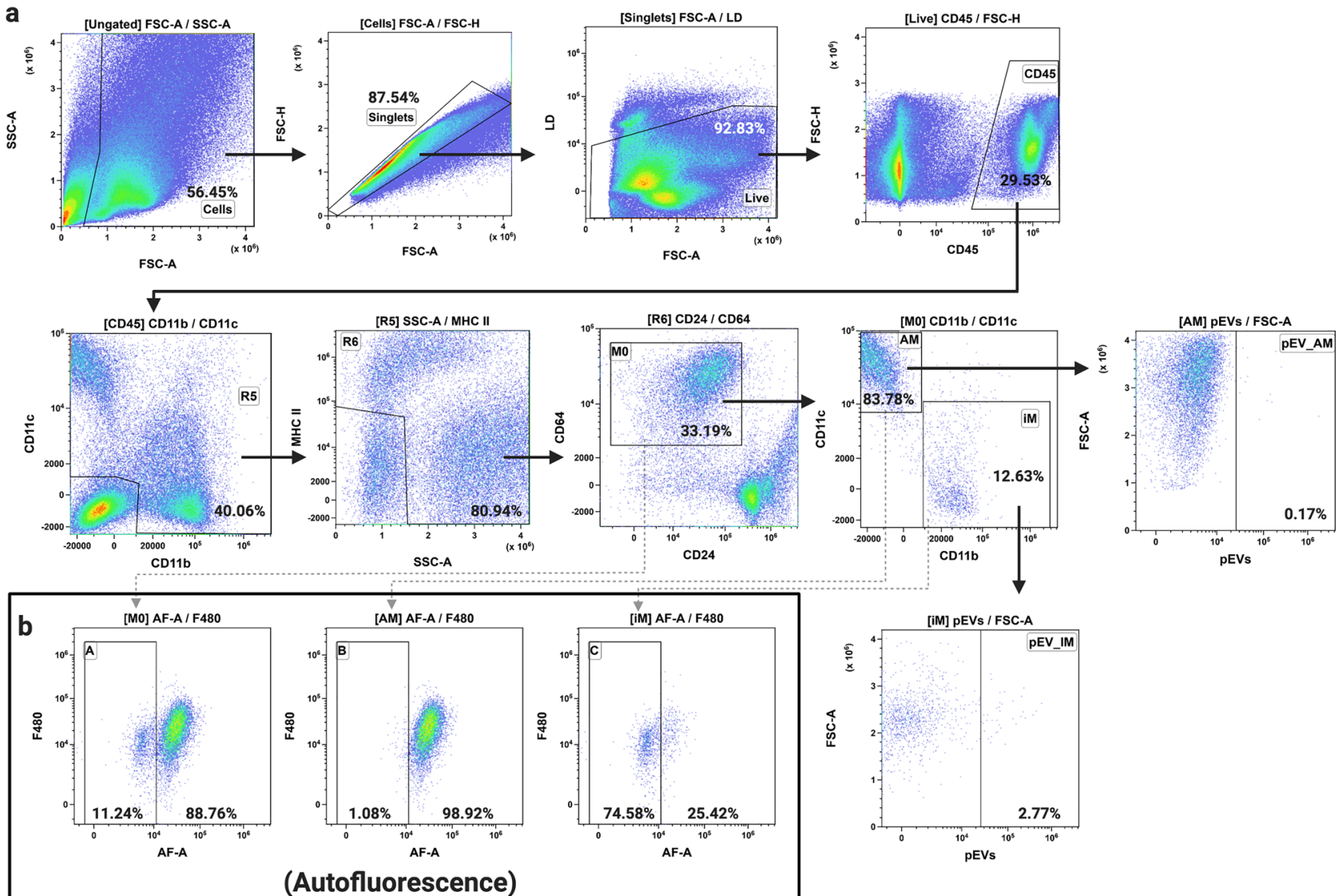

(Autofluorescence)

Figure 3. Flow cytometric analysis of pEV trafficking in murine lung. (a) Representative flow cytometry plots (of $n=7$ ) identifying macrophages (M0), alveolar macrophages (AM), and interstitial macrophages (iM) from murine lung after treatment of mice with GD14.5 placental EVs. (b) Autofluorescence signature of macrophage, alveolar macrophages, and interstitial macrophage populations; $\mathrm{n}=7$.

those mated to WT males, therefore ruling out random recombination of the $\mathrm{mT} / \mathrm{mG}$ locus in our experimental animals.

Placental EVs can induce Cre-mediated recombination in vitro.. Recombined mGFP-positive cells observed in the lungs could represent fetal cells that trafficked from the fetus/placenta to the maternal lungs (fetal microchimerism ${ }^{26-28}$. Alternatively, they could be maternal cells that underwent Cre-mediated recombination as a result of EVs carrying Cre mRNA or protein to recipient cells ${ }^{29,30}$. To test the latter possibility, we cocultured bone marrow-derived dendritic cells (BMDC) from mTmG mice with GD14.5 placental explants from CMV-Cre (colorless) or WT mice (Fig. 7a). Explants were separated from the dendritic cells by a $70 \mu \mathrm{m}$ insert such that EVs released into the medium could access the underlying BMDC. After $18 \mathrm{~h}$, placentas were removed, and BMDC were cultured for an additional 5 days to allow sufficient time for Cre recombination to occur $^{25,30}$. The BMDC were screened for recombined mGFP positive cells by flow cytometry. While no changes in mTomato expression occurred in BMDC cultured with WT placentas (Fig. 7b, left), culture with CMV-Cre placentas resulted in a downward shift of events into a new mTomato-negative/mGFP negative population and an increase in the proportion of mTomato-negative/mGFP-positive population (Fig. 7b, right).

To confirm these results, we isolated the DNA from the cocultured BMDC and performed PCR using primers designed to differentially amplify the non-recombined and recombined mTmG locus (Fig. 7c) ${ }^{31}$. We observed the mGFP amplicons in DNA isolated from BMDC cocultured with CMV-Cre, but not WT placentas (Fig. 7d). Finally, confocal microscopy conducted in parallel revealed the presence of cell-associated and non-cell-associated mGFP in BMDC co-cultured with CMV-Cre placentas (Suppl. Figure 5B, D), but not WT placentas (Suppl. Figure 5A, C), which we confirmed using immunofluorescence with an anti-GFP antibody. Interestingly, the morphology of dendritic cells co-cultured with CMV-Cre placentas revealed large clusters of cells that were not observed when the cells were co-cultured withWT placentas (Suppl. Figure 5D). Our results demonstrate a proof of concept model for identifying recipient cells that internalize placenta EVs and provides an additional mechanism for studying how placental EVs induce biological effects on recipient cells. 

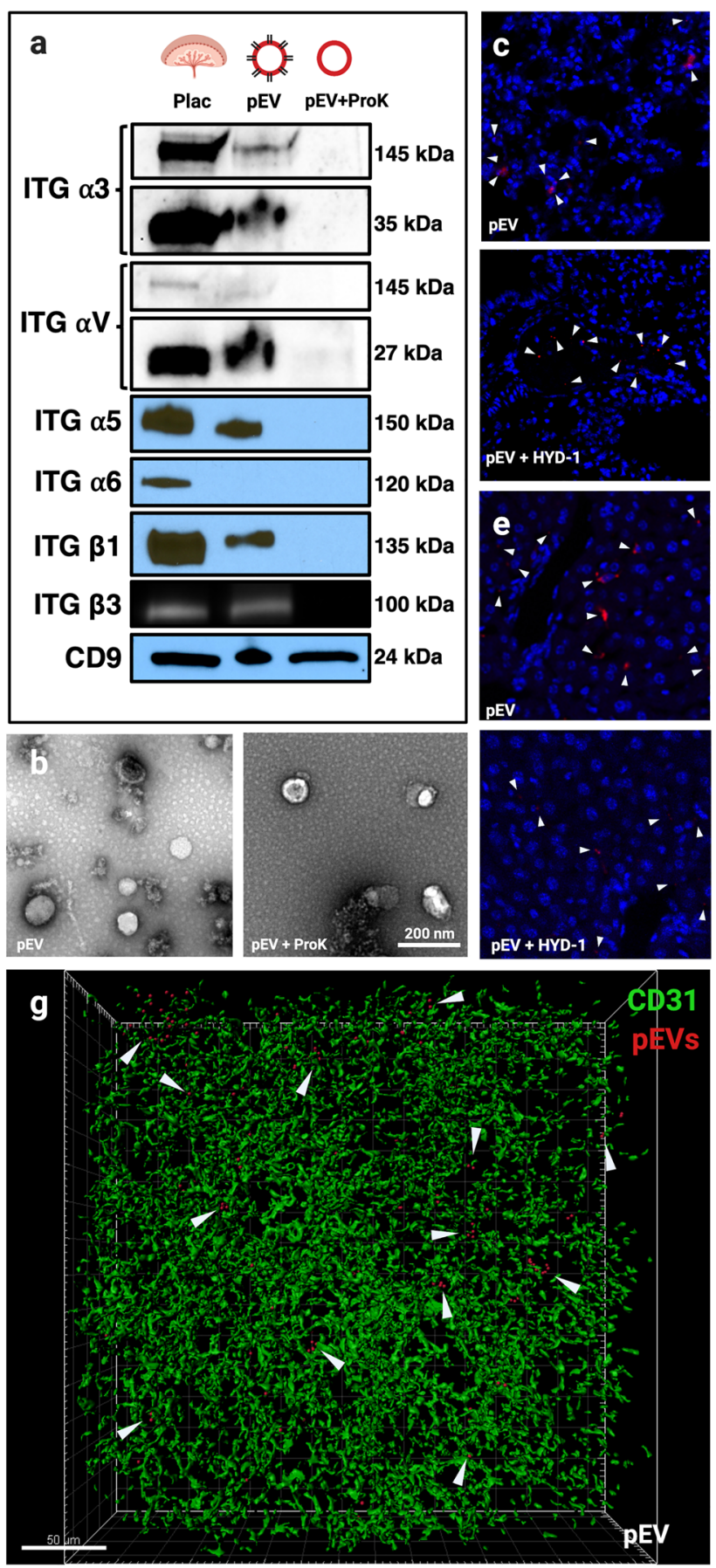
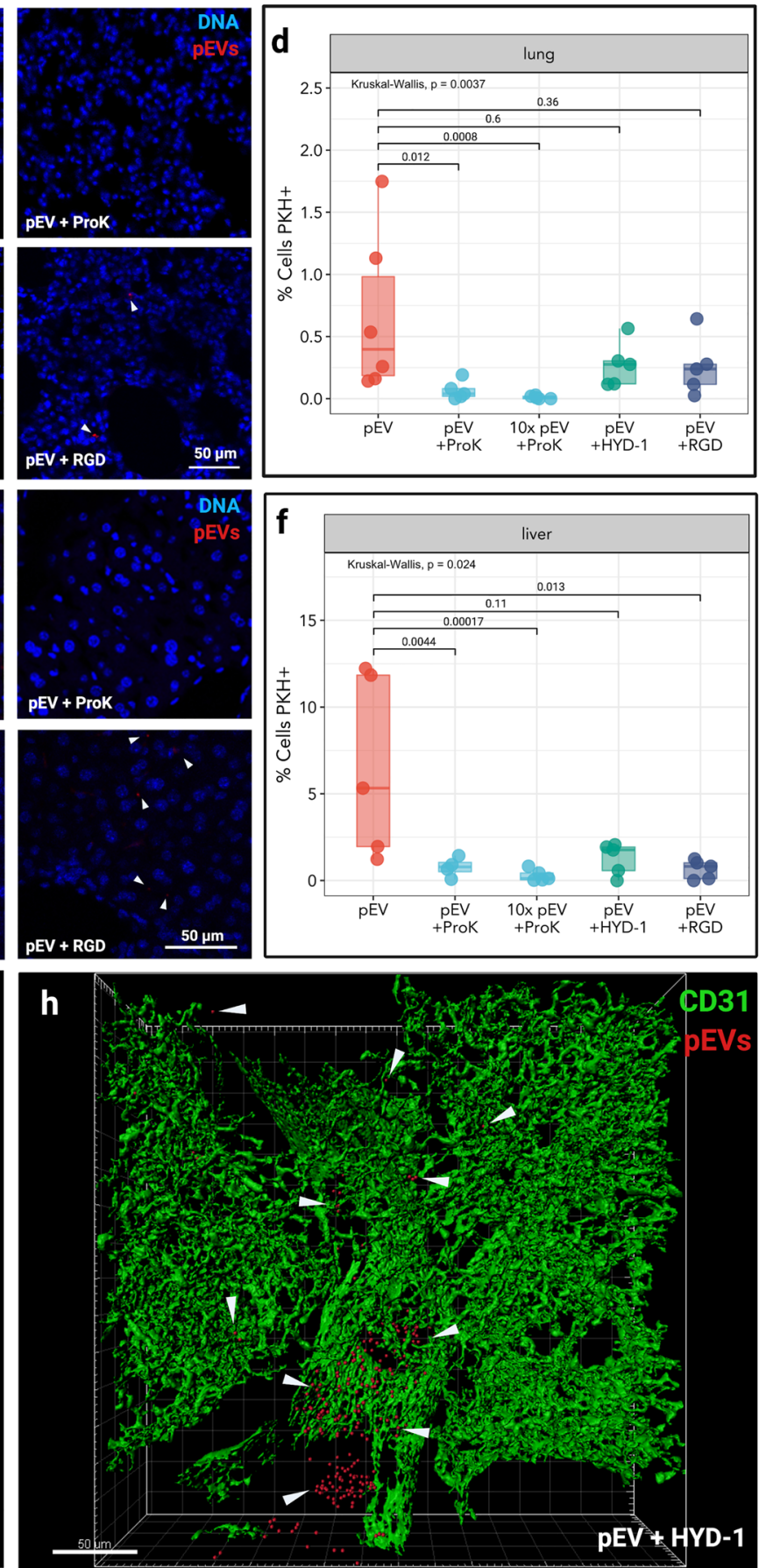

Figure 4. Integrins mediate placental EV localization to murine lung and liver. (a) Western Blot analysis of integrin expression in murine placenta, placental EVs (pEV), and placental EVs treated with proteinase $\mathrm{K}$ ( $\mathrm{pEV}+$ proK). Representative image; $\mathrm{n}=3$. (b) Transmission electron microscopy of untreated EVs (left) or EVs treated with proteinase $\mathrm{K}$ (right). Representative image of $\mathrm{n}=5$; at least five random fields were examined per replicate. (c) Confocal microscopy of murine lung after treatment of mice with GD14.5 placental EVs (upper left), proteinase K-treated EVs (upper right), or EVs pre-incubated with HYD-1 (lower left) or RGD (lower right) peptides. Images are representative of five random fields from each animal. (d) Quantification of EV positive cells in lungs treated with placental EVs; $n=5-6$ animals per treatement. (e) Confocal microscopy images of the liver after treatment of mice with placental EVs (upper left), proteinase K-treated EVs (upper right), or EVs pre-incubated with HYD-1 (lower left) or RGD (lower right) peptides. Images are representative of five random fields from each animal. (f) Quantification of EV-positive cells in liver of mice treated with placental EVs; $n=5-6$ animals per treatment. (g) CUBIC-cleared lung from mouse treated with placental EVs. White arrowheads represent placental EV foci; $n=5$. (h) CUBIC-cleared lung from mouse treated with HYD-1pretreated EVs; $\mathrm{n}=5$. 

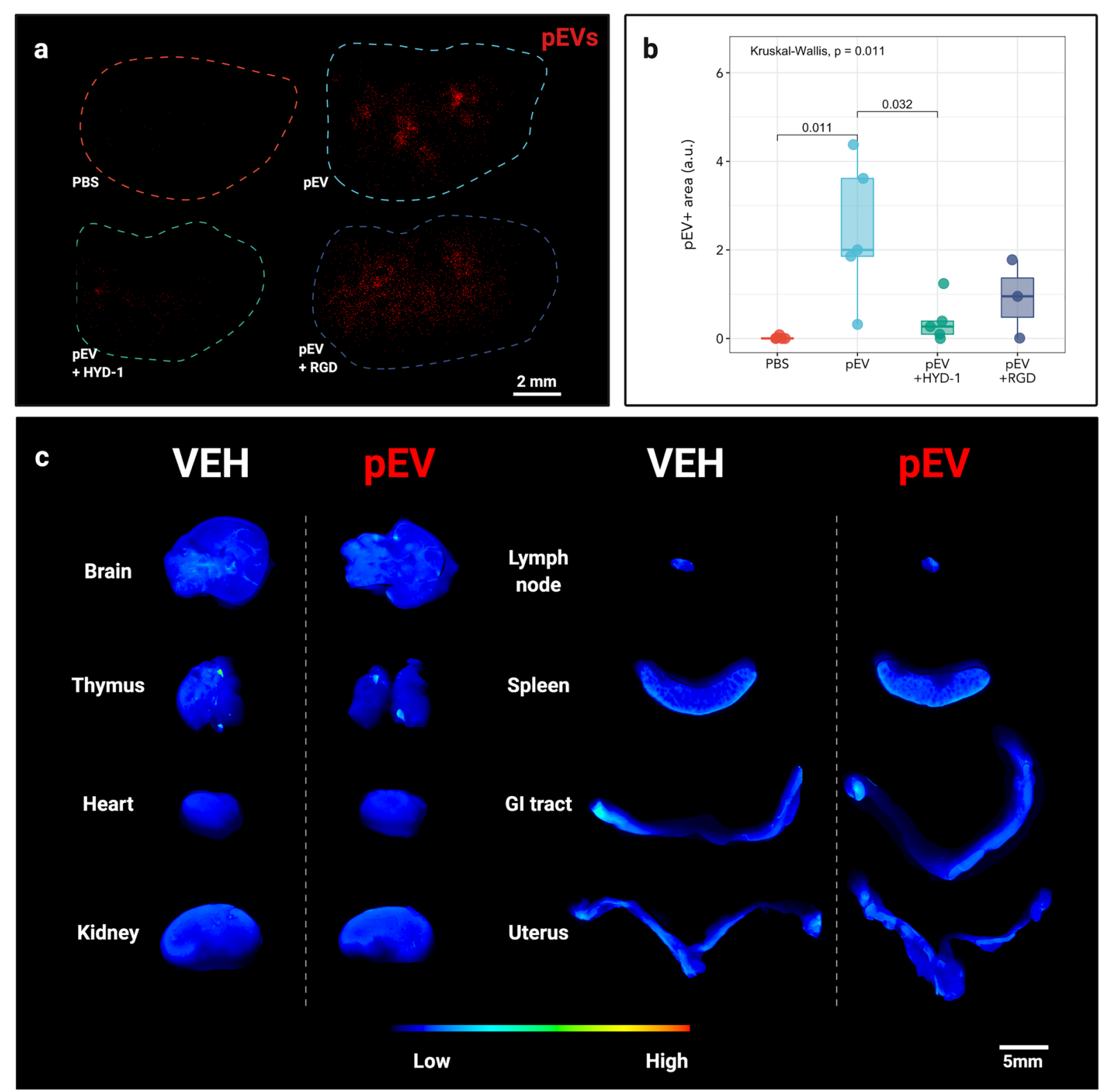

Figure 5. Integrin $\alpha 3 \beta 1$ mediates placental EV trafficking to the lung. (a) Representative near-infrared image of whole lung after in vivo treatment with labeled placental EVs after $24 \mathrm{~h}$. Image is representative of three independent experiments. (b) Quantification of NIR placental EV positive area from whole lung treated with placental EVs. a.u., arbitrary units. Each point represents one biological replicate; $n=3-7$ animals per treatment. (c) Representative whole organ NIR imaging of mice treated with control medium (VEH) or placental EVs $(\mathrm{pEV})$ after $24 \mathrm{~h}$. Representative of three independent experiments using five total animals $(\mathrm{n}=5)$.

\section{Discussion}

Since the discovery of EVs and their effects on distant cells, research interest in the role of placental EVs during pregnancy has greatly increased. The total quantity and concentration of EVs in maternal plasma rise across gestation, with the placenta contributing significantly to this increase ${ }^{5,6}$. While a number of effects of placental EVs have been suggested, few studies have attempted to quantify their biodistribution in vivo. In this study, we show that placental EVs traffic to the lung and the liver. Further, that trafficking to the lung appears to be specific to placental EVs and pregnancy, as only plasma EVs isolated from pregnant dams, but not those from non-pregnant mice, localized to the lung.

Our results align with those of Tong et al. ${ }^{17}$ who found that human placental EVs administered into mice also localize to the lung and liver. In our study, we add to these findings by using homologous adoptive transfer of murine EVs to identify the cellular targets of placental EVs in these tissues. Using multiparameter flow cytometry together with both targeted and untargeted analyses, as well as immunofluorescence microscopy, we found that murine placental EVs target lung interstitial macrophages and liver Kupffer cells. While this result is not especially surprising, it reveals for the first time the bona fide in vivo targets of EVs and aligns with earlier data showing that human trophoblast EVs are internalized by macrophages in vitro ${ }^{11}$. 

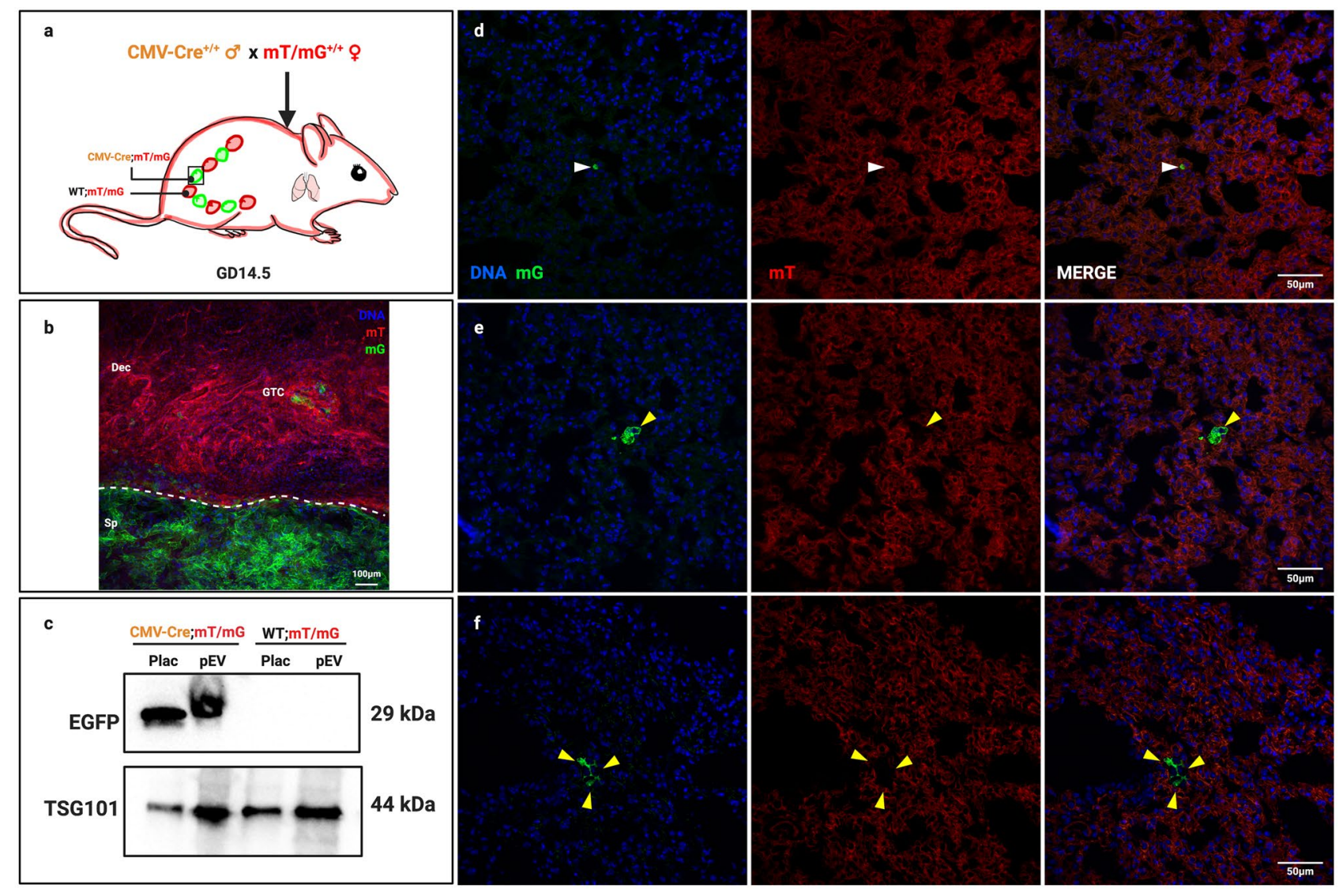

Figure 6. In Vivo Localization of mGFP in CMV-Cre Mated Maternal mT/mG Lung. (a) mT/mG females mated to a CMV-Cre males give rise to female pups ubiquitously expressing $\mathrm{mGFP}$ and male pups expressing mTomato. (b) GD14.5 utero-placental interface of a female fetus from a mT/mG x CMV-Cre mating. Fetal mGFP-expressing placental cells (Sp; spongiotrophoblast) are readily distinguishable from maternal mTomatoexpressing decidual cells (Dec); invading glycogen trophoblast (GTC) can also be seen. The dashed line demarcates the fetomaternal interface. Representative image of three independent experiments using 5 total animals $(\mathrm{n}=5)$. (c) Western blot of GD14.5 placenta (Plac) and placental EVs (pEVs) from mT/mG females mated with CMV-Cre or WT males; image is representative of four biological replicates. (d) Representative confocal image (of 5 random fields) of punctate mGFP-positive foci localization in maternal mT/mG GD14.5 lung after mating to a $\mathrm{CMV}$-Cre male. $\mathrm{N}=5$. (e,f) Confocal localization of $\mathrm{mG}+$ recombined cells in maternal $\mathrm{mT} / \mathrm{mG}$ GD14.5 lung after mating to a CMV-Cre male. Images are representative of five biological replicates. White arrows; punctate mGFP-positive foci. Yellow arrows; mGFP-positive, recombined cells.

EVs derived from Swan-71 cells, an extravillous trophoblast cell line, induce migration and proinflammatory cytokine production by cultured macrophages ${ }^{11}$. Similarly, Southcombe et al. ${ }^{32}$ showed that syncytiotrophoblast microparticles, possibly including exosomes, induce pro-inflammatory cytokine release from monocytes. While the precise role of this induction of cytokines by monocytes is uncertain, studies have supported the notion that pregnancy is associated with a shift in inflammatory environment in general, with a bias towards a proinflammatory milieu in early and late gestation, and anti-inflammatory milieu during mid-gestation ${ }^{33}$.

Whether these observations hold true for the effects of EVs on lung interstitial macrophages, which play a role homeostasis ${ }^{19,34}$, is unknown, and the impact of placental EVs on pulmonary physiology warrants investigation. Pregnancy alters maternal pulmonary function dramatically, with up to $20 \%$ increase in maternal oxygen consumption by term ${ }^{35}$, and differential susceptibility to respiratory pathology caused by influenza virus ${ }^{36,37}$; varicella virus ${ }^{38,39}$; asthma ${ }^{40}$; and cigarette smoking ${ }^{41}$. The notion that placenta- and pregnancy-associated EVs mediate these physiological and pathological adaptations to pregnancy via effects on pulmonary immune cells including macrophages is untested but intriguing.

Another important function of macrophages is antigen presentation. Maternal $\mathrm{T}$ cells are made aware of fetal antigen through indirect antigen presentation by maternal antigen presenting cells ${ }^{42}$, which may include macrophages. Maternal T cells that recognize fetal antigen do not mount an adverse immune response to fetal antigen, even when artificially stimulated with high concentrations of adjuvant ${ }^{42}$, suggesting that antigen presenting cells in pregnancy convey powerful tolerogenic signals. We and others have postulated that EVs are the source of these tolerogenic signals, possibly through cargo that include potent suppressors such as PDL1 and FASL, as well as fetal antigen itself $\mathrm{f}^{13,14,43}$. Surprisingly, our current results show minimal or no localization of placental EVs to the maternal spleen and lymph nodes, where fetal antigen recognition by maternal $\mathrm{T}$ cells occurs ${ }^{42,44}$, but 
a

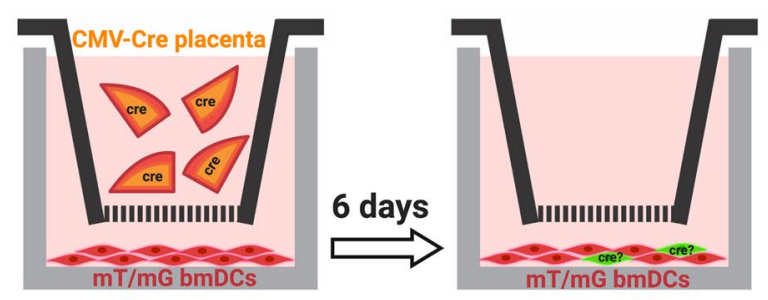

C

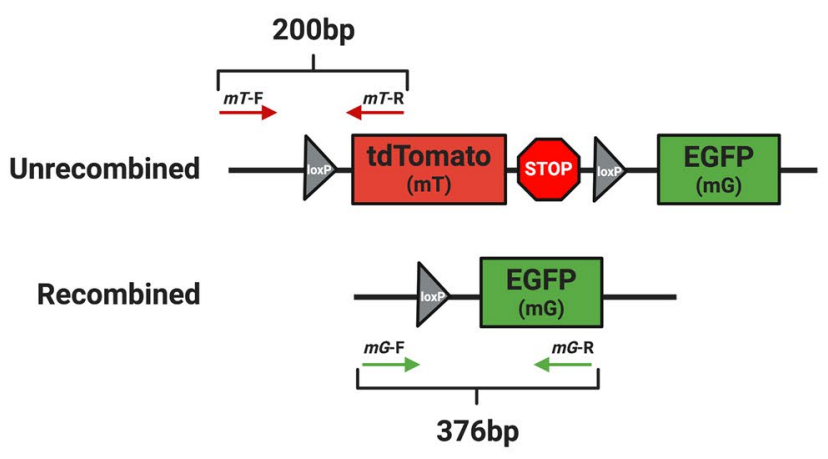

b
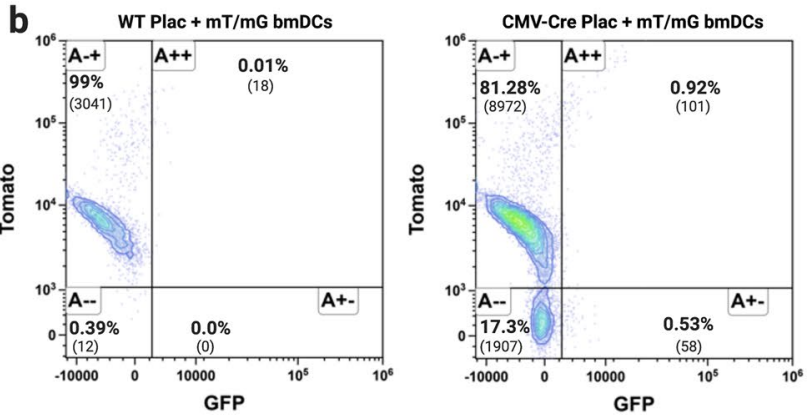

d

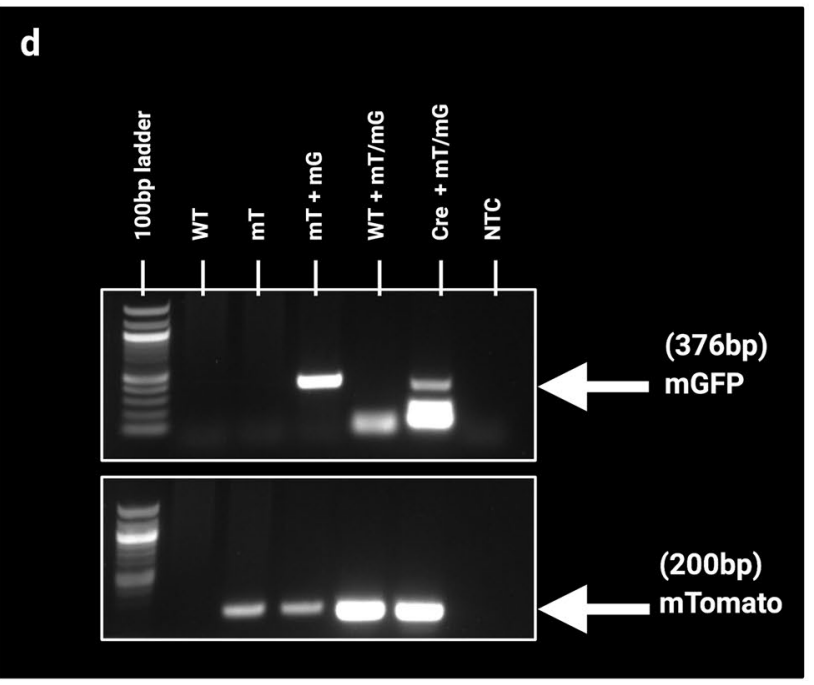

Figure 7. In Vitro Transfer of Placental Cre to Recipient Reporter Cells. (a) mT/mG BMDCs were cultured with (colorless) GD14.5 CMV-Cre placentas for $24 \mathrm{~h}$ and cultured for an additional 5 days. (b) Representative flow cytometry plots of $\mathrm{mT} / \mathrm{mG}$ BMDCs cocultured with WT (left) or CMV-Cre (right) placentas; $\mathrm{n}=1$. (c) Schematic diagram of forward (mT-F/mG-F) and reverse (mT-R/mG-R) primers for identification of unrecombined mTomato and recombined mGFP. PCR amplification of genomic DNA with these primers results in PCR products of $376 \mathrm{bp}$ for non-recombined cells and $200 \mathrm{bp}$ for recombined cells. (d) Genotyping electrophoresis gel of mGFP (top) and mTomato (bottom) loci from genomic DNA. WT: tail DNA from a wild type mouse; mT: tail DNA from an $\mathrm{mTmG}$ mouse in the absence of Cre; $\mathrm{mT}+\mathrm{mG}$ : tail DNA from a fully $\mathrm{mTmG}$ mouse; WT plac $+\mathrm{mT} / \mathrm{mG}$ : DNA from dendritic cells cocultured with WT placenta; CMV-Cre $\mathrm{plac}+\mathrm{mT} / \mathrm{mG}$ BMDC: DNA from dendritic cells cocultured with CMV-Cre placenta; NTC, no template control. Representative gel image; $\mathrm{n}=5$.

rather to lung interstitial macrophages and Kupffer cells of the liver. Lung interstitial macrophages together with resident dendritic cells are potent antigen presenting cells ${ }^{19}$. Future work can examine the role of placental EVs in antigen presentation by these macrophages as a possible mechanism for maternal immune system exposure and tolerance to fetal-placental antigens.

Our results highlight possible roles for integrins on placental EVs. The integrin profile in placental EVs did not fully recapitulate integrin expression in the placenta: integrins $\alpha 3, \alpha \mathrm{V}, \alpha 5, \beta 1$, and $\beta 3$ were expressed in both placenta and EVs, while $\alpha 6$ was found only in the placenta. This suggests that proteins are selectively loaded into placental EVs during their biogenesis, a notion supported by numerous other studies ${ }^{45}$. Additionally, removal of surface proteins, including integrins, using proteinase $\mathrm{K}$ pre-treatment disrupted the trafficking pattern of placental EVs. Further, pre-incubation of EVs with RGD peptide, which blocks ITG $\alpha 5 \beta 1$ and $\alpha$ V $\beta 3$ binding to its receptor, fibronectin, abrogated appearance of placental EVs in the liver, which is rich in fibronectin ${ }^{46}$. Similarly, HYD-1, which blocks ITG $\alpha 3 \beta 1$ binding to the pulmonary basement membrane glycoprotein laminin (LN)$5^{23,47}$, prevented entry of placental EVs into the lung. This observation was supported by 3-dimensional imaging, which highlighted association of EVs with lung endothelial cells, and confirmed that EVs remain restricted to large vessels in the lung when pre-incubated with the HYD-1 peptide.

Placental EV adhesion and extracellular matrix proteins may also play an important role in metastasis of choriocarcinoma. Trophoblast cells-the source of EVs in our studies-share many properties with cancer cells, including epithelial-to-mesenchymal transition and invasion into adjacent tissues during the physiological process of embryo implantation ${ }^{48}$. When trophoblast cells become malignant, the lung is a primary site for metastasis. In a murine xenograft model of cancer, EVs derived from metastatic breast cancer tumors selectively trafficked to the lung and liver, which served to establish a niche for future metastasis to these tissues ${ }^{21}$. Moreover, 
integrins played a major role in the selective targeting of EVs to the lung ${ }^{21}$. Thus, placental EV trafficking to the lung could play an unfortunate role in establishing a niche, parallel to that found for other metastatic tumors.

A limitation of our studies is that bolus injection of purified EVs does not recapitulate the physiological process of continuous EV release during pregnancy. We sought to address this caveat in multiple ways. First, we used a quantity of EV that mimicked quantities found during pregnancy ${ }^{6}$. Second, we administered EVs into the tail vein, reasoning that this route most closely mimics hematogenous release of placental EV in situ, as both this and the uterine vein ultimately drain into the inferior vena cava. Thus, although we were surprised that our results did not show trafficking of placental EVs to the uterus, we believe that intravenous administration simulates the hematogenous route of EVs during pregnancy.

A third way we addressed the limitations of bolus EV administration was to develop an in vivo model without the need to isolate, label and administer them. In this system, fetal tissues and EVs derived thereof express a mGFP reporter that enables their in vivo tissue and cellular targets. EVs could be detected as punctate foci within the maternal lung pregnancy, mimicking the pattern observed after intravenous injection. We did not see dramatically increased quantities of EV foci in this in vivo system, as might be expected from a continuous release from the placenta. This may be due to differences in fluorescence intensity, and therefore resolving ability, of PKH26-labeled versus GFP-expressing EVs. Nonetheless, we propose that this model is most representative date of continuous placental EV release and trafficking, and that it will allow further studies on the effects of EVs on maternal physiology in vivo.

We also identified whole cells in the lung that had recombined to express mGFP but not mTomato. Although technically these cells may produce their own EVs, we did not observe an increased concentration of EVs surrounding them, possibly due to a relatively low production of EVs by individual cells and/or spatial restriction by surrounding cells and extracellular matrix that may limit EV dispersion. Recombined mGFP-expressing cells in the maternal lung may arise from either or both of two mechanisms: genetic recombination of maternal lung cells by placental EVs, or fetal microchimerism. In our model, Cre recombinase is inherited paternally by the fetus, causing recombination of the mTmG locus that switches cells from red to green fluorescence. Using similar models, others have shown that Cre mRNA is carried by exosomes secreted by cancer cells, and that these exosomes could thus induce recombination of neighboring or distant cells ${ }^{30}$. To test whether this is possible in principle, we used a model system in which red fluorescent $\mathrm{mTmG}$ dendritic cells were co-cultured with CMVCre placentas. Data generated by immunofluorescence microscopy, flow cytometry, and genetic analysis support the notion that shed vesicles from the explants induced recombination in the dendritic cells.

A second possible explanation for our observation of recombined cells in the mother is fetal microchimerism. Fetal cells that traffic into maternal tissues was first described with the presence of fetal trophoblast cells residing in the maternal lung over 125 years ago ${ }^{49}$ and has been confirmed by numerous studies since ${ }^{26,50,51}$. Fetal microchimerism is persistent: male fetal progenitor cells are detectable in maternal blood for decades postpartum ${ }^{50}$. Murine models suggest that fetal nucleated cells are detectable in maternal lung ${ }^{26,27}$; the tissue specificity of microchimerism may depend on maternal physiological and pathological conditions ${ }^{52}$. To elucidate whether genetic recombination of maternal cells and/or fetal microchimerism is responsible for the appearance of recombined cells in our model, ongoing studies in our lab seek to identify Cre recombinase protein or mRNA in EVs and will use in vivo approaches to analyze directly whether placental EVs can induce genetic changes in maternal cells.

Collectively, we have established that placental EVs preferentially traffic to maternal pulmonary interstitial macrophages and Kupffer cells in vivo. We demonstrate that integrin $\alpha 3 \beta 1$ is necessary for localization to the lung interstitial tissue, and that integrins $\alpha 5 \beta 1$ and $\alpha \mathrm{V} \beta 5$ are necessary for localization to the liver. Future work will seek to identify how placental EVs influence maternal interstitial macrophages and Kupffer cell function, as well as the physiology of the lung and liver, during pregnancy. Additionally, we demonstrate a new model for further expanding the field of understanding placental EV interactions in vivo and provide a framework for visualizing maternal-fetal interactions without the use of exogenous purification and labeling, providing a strong advantage to traditional methods of tracking placental EV kinetics in vivo in a manner consistent with their physiological release across pregnancy.

\section{Methods}

Animal experiments. All animal experiments were conducted in accordance with approved protocols based on guidelines and regulations of the AAALAC-accreted vivarium at Michigan State University and approved by their Institutional Animal Care and Use Committee (protocol no. 201800176). Wildtype C57BL/6J,

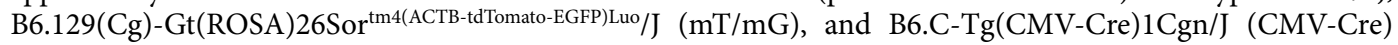
mice were purchased from the Jackson Laboratory (Bar Harbor, ME) and housed in temperature-controlled, 12:12 h light/dark cycle rooms with food and water available ad libitum. Mice used in experiments were aged 6-12 weeks and timed matings were performed, in which the presence of a vaginal plug was designated gestational day (GD) 0.5 . For plasma isolation mice were anesthetized with $3 \%$ isoflurane infused with oxygen ( $2 \mathrm{l} /$ $\mathrm{min}$ ), and. whole blood was collected by cardiac puncture into EDTA-containing tubes. For injection of EVs and subsequent tissue harvesting, mice were anesthetized and EVs administered via the caudal vein, and tissues were harvested after $30 \mathrm{~min}$ or $24 \mathrm{~h}$. All mice were euthanized under anesthesia by cervical dislocation and bilateral pneumothorax.

Materials and reagents. Chemicals, reagents, and kits were obtained from Thermo-Fisher (Rockford, IL) or Sigma-Aldrich (St. Louis, MO) unless otherwise stated. Filters and membranes were obtained from Millipore (Burlington, MA), and tissue culture materials were purchased from Corning (Corning, NY). Antibodies and other key reagents are listed in Table 1 . All figures were created using www.BioRender.com. 


\begin{tabular}{|c|c|c|c|c|}
\hline Reagent type (species) or resource & Designation & Source or reference & Identifiers & Additional information \\
\hline $\begin{array}{l}\text { Strain, strain background ( } M . \\
\text { musculus) }\end{array}$ & WT & Jackson Laboratory & Jax:000664 & \\
\hline $\begin{array}{l}\text { Strain, strain background ( } M . \\
\text { musculus) }\end{array}$ & CMV-Cre & Jackson Laboratory & Jax:006054 & \\
\hline $\begin{array}{l}\text { Strain, strain background ( } M . \\
\text { musculus) }\end{array}$ & $\mathrm{mTmG}$ & Jackson Laboratory & Jax:007676 & \\
\hline Software, algorithm & Imaris & Oxford imaging & & \\
\hline Software, algorithm & CellProfiler 3.0 & https://cellprofiler.org/ & & \\
\hline Software, algorithm & Fiji/imagej & https://fiji.sc/ & & \\
\hline Software, algorithm & Cytofkit & Chen et al. ${ }^{56}$ PMID: 27662185 & $\begin{array}{l}\text { https://github.com/JinmiaoChenLab/ } \\
\text { cytofkit }\end{array}$ & \\
\hline Commercial assay or kit & PKH26 & Sigma-Aldrich & Sigma:MINI26-1KT & EV labeling \\
\hline Commercial assay or kit & $\begin{array}{l}\text { DiR'; DiIC18(7) } \\
\text { (1,1'-dioctadecyl-3,3,3',3'- } \\
\text { Tetramethylindotricarbocyanine } \\
\text { Iodide) }\end{array}$ & Thermo Fisher & Fisher:D12731 & EV labeling \\
\hline Commercial assay or kit & $\begin{array}{l}\text { Quick DNA/RNA isolation miniprep } \\
\text { kit }\end{array}$ & Zymo & Zymo:D7003 & \\
\hline Recombinant protein & Proteinase $\mathrm{K}$ & & & $10 \mu \mathrm{g} / \mathrm{ml}$ \\
\hline Peptide & RGD & Sigma-Aldrich & Sigma:A8052-5MG & $>97 \%$ purity, $0.6 \mu \mathrm{M}$ \\
\hline Peptide & HYD-1 & Genscript & Genscript:KIKMVISWKG & $>98 \%$ purity, $0.6 \mu \mathrm{M}$ \\
\hline Antibody & $\begin{array}{l}\text { Alexafluor } 488 \text { anti-LYVE1 (rat } \\
\text { monoclonal) }\end{array}$ & Invitrogen & Invitrogen:53-0443-80 & $(1: 100)$ \\
\hline Antibody & $\begin{array}{l}\text { Alexafluor } 647 \text { anti-CD68 (rat } \\
\text { monoclonal) }\end{array}$ & BioLegend & BioLegend:137004 & $(1: 100)$ \\
\hline Antibody & $\begin{array}{l}\text { Alexafluor } 647 \text { anti-GFP (rat mono- } \\
\text { clonal) }\end{array}$ & BioLegend & BioLegend:338006 & $(1: 100)$ \\
\hline Antibody & Anti-CD31 (rat monoclonal) & BioLegend & BioLegend:102402 & $10 \mu \mathrm{g} / \mathrm{ml}$ \\
\hline Antibody & $\begin{array}{l}\text { Alexafluor } 488 \text { anti-Rat IgG (goat } \\
\text { polyclonal) }\end{array}$ & Invitrogen & Invitrogen: A-11006 & $10 \mu \mathrm{g} / \mathrm{ml}$ \\
\hline Antibody & APC-Cy7 anti-F4/80 & BioLegend & BioLegend:123118 & $(1: 100)$ \\
\hline Antibody & Live/Dead-Yellow & Thermo Fisher & Thermo Fisher: & $(1: 1000)$ \\
\hline Antibody & PE-Cy5 anti-CD45 & BioLegend & BioLegend:103110 & $(1: 200)$ \\
\hline Antibody & BV421 anti-CD11c & BioLegend & BioLegend:117343 & $(1: 200)$ \\
\hline Antibody & PerCp-Cy5.5 anti-CD11b & BioLegend & BioLegend:101228 & $(1: 200)$ \\
\hline Antibody & PE-Cy7anti-MHCII & BioLegend & BioLegend:107630 & $(1: 600)$ \\
\hline Antibody & FITC anti-CD64 & BioLegend & BioLegend:139316 & $(1: 125)$ \\
\hline Antibody & APC anti-CD24 & BioLegend & BioLegend:138506 & $(1: 100)$ \\
\hline Antibody & Anti-TSG101 (rabbit polyclonal) & Abcam & Abcam: ab30871 & $(1: 1000)$ \\
\hline Antibody & Anti-CD9 (mouse monoclonal) & Invitrogen & Invitrogen: 10626D & $1 \mu \mathrm{g} / \mathrm{ml}$ \\
\hline Antibody & Anti-ITG alpha 3 (goat polyclonal) & R\&D & R\&D: AF2787 & $0.1 \mu \mathrm{g} / \mathrm{ml}$ \\
\hline Antibody & $\begin{array}{l}\text { Anti-ITG alpha V (rabbit mono- } \\
\text { clonal) }\end{array}$ & Abcam & Abcam: ab124968 & $(1: 1000)$ \\
\hline Antibody & Anti-ITG alpha 5 (rabbit monoclonal) & Cell Signal Technologies & CST: & $(1: 1000)$ \\
\hline Antibody & Anti-ITG alpha 6 (rabbit monoclonal) & Cell Signal Technologies & CST: & $(1: 1000)$ \\
\hline Antibody & Anti-ITG beta 1 (rabbit monoclonal) & Cell Signal Technologies & CST: & $(1: 1000)$ \\
\hline Antibody & Anti-ITG beta 3 (rabbit monoclonal) & Cell Signal Technologies & CST:13166 & $(1: 1000)$ \\
\hline Antibody & Anti-EGFP (goat polyclonal) & Origene & Origene:TA150095 & $(1: 2000)$ \\
\hline Antibody & HRP anti Rabbit IgG (goat polyclonal) & Cell Signal Technologies & CST: 7074 & $(1: 2000)$ \\
\hline Other & CUBIC1 & Susaki et al. ${ }^{24}$, PMID: 24746791 & & \\
\hline Other & CUBIC2 & Susaki et al. ${ }^{24}$, PMID: 24746791 & & \\
\hline Other & Mounting medium with DAPI & Vector Labs & Vector & 25-50 ul per coverslip \\
\hline
\end{tabular}

Table 1. Key resources. Information includes species and strains of animals used in this study, software, kits, antibodies, and reagents.

Placental explant culture. EV-free media (RPMI 1640, $50 \mu \mathrm{M} \beta$-mercaptoethanol, $100 \mu \mathrm{g} / \mathrm{ml}$ Penicillin/ Streptomycin, $1 \mathrm{mM}$ Sodium pyruvate, $10 \%$ fetal bovine serum) was ultracentrifuged at $100,000 \times g$ for $18 \mathrm{~h}$ at $4{ }^{\circ} \mathrm{C}$ and sterilized through a $0.22 \mu \mathrm{m}$ filter. Placental explant culture was performed as previously described ${ }^{53}$; briefly, individual GD14.5-16.5 placentas were cut into four pieces and placed in $74 \mu \mathrm{m}$ mesh $15 \mathrm{~mm}$ net well inserts in a 12-well plate filled with $3 \mathrm{ml}$ of EV-depleted media for $18 \mathrm{~h}$ at $37^{\circ} \mathrm{C}, 5 \% \mathrm{CO}_{2}$. To determine optimal culture conditions, viability of tissue explants was assessed by a board-certified veterinary pathologist (DA) by 
examination of hematoxylin and eosin stained sections using a double blinded scoring system for placental tissue necrosis, using a scale of 0 (no necrosis) to 5 (complete necrosis). Following culture, the culture supernatant was centrifuged twice at $500 \times g$ and $2000 \times g$ for $15 \mathrm{~min}$ to pellet cells and cellular debris, respectively. Supernatant was filtered through a $0.22 \mu \mathrm{m}$ PVDF membrane filter and concentrated to a volume of $500 \mu \mathrm{l}$ at $3320 \times g$ using $100 \mathrm{kDa}$ molecular weight cut off Vivaspin centrifugal ultrafiltration columns (Sartorius, Stonehouse, UK). The resulting filtrate was stored at $-80^{\circ} \mathrm{C}$ until processed for $\mathrm{EV}$ isolation.

To ensure EVs were isolated from high quality tissue, we first tested the viability of the cultured explants under various conditions, as well as the quantity (Suppl. Figure 2A, B) and quallity (Suppl. Figure 2C, D) of EVs. We saw no significant differences in necrosis or EVs between freshly isolated and cultured placentas, although the latter exhibited more variation. We observed the highest yield of EVs from placentas cultured for 24-h at ambient oxygen, and therefore used these conditions for further studies. For low oxygen culture, tissues were placed in a sealed gas chamber and purged of ambient air and charged with a gas mixture containing $8 \%$ oxygen, $5 \%$ carbon dioxide before being sealed and placed in an incubator.

Extracellular vesicle isolation and labeling. Extracellular vesicles were isolated from plasma and concentrated supernatant of explant cultures using qEVsingle or qEVoriginal size exclusion chromatography columns (Izon Sciences, Medford, MA) following the manufacturer's instructions. Briefly, plasma or supernatant were loaded onto columns equilibrated with PBS and fractions 6-8 or 7-9 (200 $\mu$ l for qEVsingle, $500 \mu \mathrm{l}$ for qEVoriginal columns, respectively) were collected and concentrated to $50 \mu \mathrm{l}$ using Amicon Ultra $410 \mathrm{kDa}$ centrifugal filters for further analysis.

Purified EVs were labeled with PKH26 lipophilic red fluorescent dye (Sigma-Aldrich, St. Louis, MO) using the manufacturer's instructions. Briefly, purified EVs or PBS (negative control for injection) were diluted in Diluent $\mathrm{C}$ and pipetted into ultracentrifuge tubes containing PKH26 dye and Diluent C. The labeling reaction was stopped after $5 \mathrm{~min}$ with the addition of $10 \% \mathrm{BSA}$ and EV-depleted culture medium. To eliminate excess unbound dye, $0.971 \mathrm{M}$ sucrose was pipetted beneath the labeled EVs and centrifuged for $1.5 \mathrm{~h}$ at $150,000 \times g$ at $4{ }^{\circ} \mathrm{C}$ as described ${ }^{54}$. The labeled EVs were washed and concentrated with Amicon Ultra $410 \mathrm{kDa}$ centrifugal filters as described above. Prior to administration, we separated any remaining free dye from labeled EV by centrifugation over a sucrose gradient; as a negative control, $\mathrm{PKH} 26$ dye alone was processed and administered in parallel.

For experiments in which whole organ imaging was performed, purified EVs were labeled with $0.246 \mathrm{mM}$ [DiIC18(7) (1,1'-dioctadecyl-3,3,3',3'-Tetramethylindotricarbocyanine Iodide)] DiR for 3 min and ultracentrifuged on a sucrose cushion as described above. After centrifugation, the labeled vesicles were loaded on IZON single columns and fractions 6-8 were collected and concentrated with $10 \mathrm{kDa}$ concentrators as described above and used for downstream analysis.

Nanoparticle tracking analysis. EVs were quantified using NanoSight NS300 (Malvern Panalytical,Westborough, MA) as described previously with modifications ${ }^{6}$. Briefly, concentrated samples were diluted 1:500-2000 in PBS and loaded into a $1 \mathrm{ml}$ syringe at a flow rate of 50. For each sample, five 20-s videos were recorded at camera level 12 and a detection threshold of 4 before data raw data were analyzed with the tidyNano R package ${ }^{6}$.

Transmission electron microscopy. For transmission electron microscopy, concentrated EVs were fixed with $4 \%$ paraformaldehyde in PBS and placed on formvar-carbon coated grids and counterstained with $2.5 \%$ glutaraldehyde and $0.1 \%$ uranyl acetate in PBS. Negative TEM was performed on a JEOL100CXII (JEOL, Peabody, MA).

Proteinase $\mathrm{K}$ and inhibitory peptide treatment. For proteinase $\mathrm{K}$ treatment, placental EVs were treated with $10 \mu \mathrm{g} / \mathrm{ml}$ proteinase $\mathrm{K}$ in PBS; for controls, proteinase $\mathrm{K}$ was added to PBS alone. Samples and controls were incubated for $10 \mathrm{~min}$ at $37^{\circ} \mathrm{C}$ before heat inactivation at $65^{\circ} \mathrm{C}$ for $10 \mathrm{~min}$ followed by addition of $100 \mu \mathrm{M}$ phenylmethylsulfonyl fluoride (PMSF) protease inhibitor.

For inhibitory peptide experiments, RGD peptide was purchased from Sigma-Aldrich and HYD-1 (KIKMVISWKG) was synthesized (Genscript, Piscataway, NJ). All reagents were $95 \%$ or greater purity and resuspended in deionized water, aliquoted, and frozen. Labeled pEVs were incubated with $0.6 \mu \mathrm{M}$ of inhibitory peptide and incubated at $37^{\circ} \mathrm{C}$ for $30 \mathrm{~min}$ before intravenous administration into recipient mice.

$\mathrm{mT} / \mathrm{mG}$ BMDC explant co-culture. Bone marrow derived dendritic cells (BMDC) were prepared from $\mathrm{mT} / \mathrm{mG}$ mice as described with modifications $\mathrm{s}^{55}$. Ten million bone marrow cells/well were plated overnight in medium containing $20 \mathrm{ng} / \mathrm{ml}$ murine GM-CSF(BioLegend, San Diego, CA), replenishing the medium daily for 3 days, then culturing for an additional 3 days before downstream analysis.

For co-culture with placental explants, 100,000 BMDC were seeded in 12-well plates, and CMV-Cre or WT placentas were added on Netwell inserts as described above. Following overnight culture, the placentas were removed, and the BMDC were cultured for an additional 5 days to allow for sufficient time for recombination ${ }^{25}$, replenishing the media every other day. For microscopy, BMDC were seeded onto coverslips before placental co-culture, then fixed with 4\% PFA and stained with 1:5000 rabbit anti-EGFP (Origene) and anti-rabbit AF647 $(10 \mu \mathrm{g} / \mathrm{ml})$ following culture. Coverslips were mounted with mounting medium containing DAPI (Vector) on glass slides and imaged on a Nikon A1 Confocal microscope fitted with a $20 \times, 40 \times$, or $60 \times$ objective. 
Western blot. For protein analysis, placental tissues or size exclusion column fractions were stored at $-80^{\circ} \mathrm{C}$ until downstream analysis. Tissues were homogenized in RIPA buffer supplemented with $2 \mu \mathrm{g} / \mathrm{ml}$ aprotinin, $5 \mu \mathrm{g} / \mathrm{ml}$ leupeptin, and $1 \mathrm{mM}$ phenylmethylsulfonyl fluoride (PMSF). Protein was quantified using a Qubit 3 fluorometer (Life Technologies, Carlsbad, MA). Samples were heat-denatured at $98{ }^{\circ} \mathrm{C}$ for 15 min in RIPA buffer containing dithiothreitol (DTT). Denatured samples $(10 \mu \mathrm{g} / \mathrm{lane})$ were run on precast $4-20 \%$ SDS-PAGE gels (Bio-Rad, Hercules, CA) and protein was transferred onto PVDF or nitrocellulose membranes. Membranes were blocked for 20-30 min in SuperBlock solution (ThermoFisher, Rockland, IL) at room temperature before overnight incubation at $4{ }^{\circ} \mathrm{C}$ in $3 \%$ skim/tris buffered saline (TBS) milk with anti TSG101, anti-ITG alpha V (Abcam), anti-CD9 (Thermo Fisher), anti-ITG alpha 3, anti-ITG alpha 5, anti-ITG alpha 6, anti-ITG beta 1, antiITG beta 3 (Cell Signal Technologies), or anti EGFP (Origene) primary antibody. Membranes were washed three times for 5 min in TBS with $0.05 \%$ Tween-20 (TBS-T) at RT and incubated with 1:2000 horseradish peroxidase (HRP) conjugated goat anti-rabbit secondary antibody for $1 \mathrm{~h}$ at RT. Membranes were washed three times with TBS-T for 5 min and incubated with SuperSignal PicoPlus chemiluminescent substrate for 5 min before being imaged on a digital iBright membrane imager.

Fluorescence/immunofluorescence microscopy and EV quantification in tissues. Tissues were harvested from mice were fixed in 4\% paraformaldehyde (PFA) [Sigma-Aldrich, St. Louis, MO] in PBS and 30\% sucrose in PBS at $4{ }^{\circ} \mathrm{C}$ overnight before being frozen with isopentane and liquid nitrogen in Tissue Tek O.C.T embedding compound (Sakura Finetek, Torrance, CA). For fluorescence microscopy, $5 \mu \mathrm{m}$ tissue cryosections were mounted with DAPI nuclear stain and imaged on a Nikon Eclipse Ti epifluorescent microscope fitted with a $20 \times$ NA 0.5 plan fluor objective using Nikon NIS-Elements AR 4.40 software. To quantify EV in tissues, five random fields were imaged, and individual color channels processed using ImageJ. PKH26 and nuclei counts were computationally quantified using a custom pipeline developed in CellProfiler 3.0 software. For immunofluorescence microscopy, slides were stained with BioLegend antibodies and mounted with mounting medium with DAPI.

Tissue clearing and immunofluorescence analysis. For clearing tissues prior to 3D imaging, paraformaldehyde-fixed organs were embedded in $2 \%$ agarose. Sections $(200 \mu \mathrm{m})$ were obtained using a Leica VT1200 S vibratome (Leica, Buffalo Grove, IL), placed in 12-well plates, and permeabilized in 0.05\% tween 20. Sections were blocked in $10 \%$ SuperBlock solution for $4-12 \mathrm{~h}$, and stained with antibodies at $37^{\circ} \mathrm{C}$ in an orbital shaker for $24 \mathrm{~h}$. DAPI $(10 \mathrm{ug} / \mathrm{ml})$ was added to the solution and sections were incubated for an additional $24 \mathrm{~h}$. After a 12-h wash, secondary antibody was added overnight at $37^{\circ} \mathrm{C}$, and samples were again washed for $12 \mathrm{~h}$. Samples were then immersed in 2-3 $\mathrm{ml}$ of CUBIC1 solution and incubated overnight at $37^{\circ}$ before being washed briefly with PBS before immersed with 2-3 ml of CUBIC2 solution for $12 \mathrm{~h}^{24}$.

For traditional immunofluorescence, paraformaldehyde-fixed tissues were placed in $30 \%(\mathrm{~W} / \mathrm{V})$ sucrose solution in PBS overnight before being embedded in optimal cutting tissue (O.C.T.) compound (Sakura Finetek, Torrance, CA) and freezing. Cryosections $(7 \mu \mathrm{m})$ were placed onto charged glass slides, blocked with $10 \%$ SuperBlock and $3 \%$ goat serum, and incubated overnight at $4{ }^{\circ} \mathrm{C}$ with rabbit anti-EGFP followed by goat anti-rabbit conjugated to Alexa fluor 647. Slides were washed three times with $0.05 \%$ Tween PBS (PBS-T) for 5 min, and sections were mounted in DAPI-containing medium (Vector, Burlingame, CA).

All tissues and cells were imaged on a Nikon A- 1 confocal microscope fitted with a $20 \times$ and $40 \times$ oil objectives lenses and images were processed using FIJI image analysis software. Images of the $200 \mu \mathrm{m}$ vibratome sections were also processed using the Imaris v9.2 software (Bitplane) for 3D reconstruction of vasculature and EV localization. 3D mesh of CD31 positive vasculature was created using the Surface plugin and background subtraction was applied. Placental EVs were detected using the Spots plugin based on PKH26 labeling. The diameter of EV was estimated to be $0.75 \mathrm{um}$ in the XY slice for creating spots. Images were taken using the Snapshots function and $3 \mathrm{D}$ Video was generated using the animation function of the software.

Flow cytometry. Lungs were minced and incubated in digestion buffer consisting of RPMI 1640, 0.05\% liberase TH (Roche), and 100U/ml DNase1 (Sigma-Aldrich, St. Louis, MO) for $1 \mathrm{~h}$ at $37^{\circ} \mathrm{C}$. Larger tissue pieces were further dispersed by passing through a $3 \mathrm{ml}$ syringe with a 27 gauge needle and cells were passed through a 70 micron nylon cell strainer (Falcon, Tewksbury, MA). The enzymatic digestion was quenched with $5 \%$ bovine serum albumin in PBS and cells were pelleted at $500 \times \mathrm{g}$ for $5 \mathrm{~min}$ before treatment with ACK lysis buffer ( $150 \mathrm{mM}$ $\mathrm{NH} 4,10 \mathrm{mM} \mathrm{KHCO} 3,0.1 \mathrm{mM} \mathrm{Na} 2 \mathrm{EDTA}$, in distilled water) to lyse red blood cells. Cells were resuspended in FSB ( $2 \%$ fetal bovine serum and $0.01 \%$ sodium azide in PBS), and staining was performed in U-bottom 96 well plates. Cells were incubated with Live/Dead Yellow (1:1000) followed by Fc block (1:200; BioLegend, San Diego, CA), and stained with antibody cocktails for $30 \mathrm{~min}$ on ice. Cells were washed twice and fixed with $1 \%$ PFA in flow staining buffer. Spectral flow cytometry was performed on an Aurora (Cytek BioSciences, Fremont, CA) with eUltracomp compensation beads used for single color controls. Events (100,000-200,000) were collected for each well and analysis was done using Kaluza 2.1 software (Beckman Coulter, Brea, CA). Flow cytometry $\mathrm{t}$-distributed stochastic neighbor embedding ( $\mathrm{t}$-SNE) analysis was performed using the Cytofkit $\mathrm{R}$ package ${ }^{56}$ with default settings.

To characterize BMDC expression of mTomato or mGFP fluorescence, co-cultured dendritic cells were removed from culture plates with cell scrapers and stained with live/dead yellow viability stain (L34959, Thermo Fisher, Waltham, MA) for 15 min on ice. Cells were then blocked with TruStain FcX block (Biolegend, San Diego, CA) for 10 min and stained with anti-CD45, CD11c, and MHC -II antibodies (BioLegend, San Diego, CA) as described above. Single color controls were made with individual antibodies along with eUltracomp beads (Thermo Fisher, Waltham, MA), and spleen cells from mTomato and mGFP mice were used as endogenous 
fluorescence controls. Stained cells were analyzed by an Aurora spectral flow cytometer (CytekBio, Fremont, $\mathrm{CA}$ ), and data were analyzed by Kaluza flow cytometry software (Beckman Coulter, Pasadena, CA).

Whole organ imaging. To quantify EV localization in whole organs, $2.5 \times 10^{10} \mathrm{GD} 14.5$ DiR labeled pEVs were injected intravenously into NP female mice. After $24 \mathrm{~h}$, mice were euthanized and the lung, liver, spleen, thymus, brain, uterus, spleen, lymph node, kidney, and heart were harvested and fixed in 4\% PFA in PBS for four hours before being stored in PBS. Whole organs were imaged on a LiCor odyssey infrared imager on manual scan mode (21 microns) under automatic acquisition settings (LiCor BioSciences, Lincoln, NE). Raw tifs were analyzed using Fiji and converted to 8 bit before mean intensity was measured in each tissue.

Recombination genotyping. To detect genomic recombination in co-cultured $\mathrm{mT} / \mathrm{mG}$ dendritic cells, DNA was isolated with a Quick DNA/RNA miniprep kit (D7005, Zymo, Irvine, CA) following the manufacturer's instructions. DNA was amplified using a modified two-step polymerase chain reaction protocol targeting the unrecombined mTomato locus or recombined $\mathrm{mGFP}$ locus in $\mathrm{mT} / \mathrm{mG}$ reporter (Fig. $7 \mathrm{c}$ ) mice ${ }^{31}$. The mTomato locus was amplified with mT-F 5'-GCAACGTGCTGGTTATTGTG-3' and mT-R 5'-TGATGACCTCCTCTC CCTTG-3' primers, yielding a $200 \mathrm{bp}$ amplicon. The mGFP locus was amplified with mG-F 5'-GTTCGGCTT CTGGCGTGT-3' and mG-R5'-TGCTCACGGATCCTACCTTC- 3' primers, yielding a 376 amplicon. Genomic DNAfrom dendritic cells was amplified for 35 cycles, and $3 \mu \mathrm{l}$ of the PCR product was used as the template for a second round of PCR. Genomic tail DNA from WT, mTomato, and mGFP mice were used as positive and negative controls for both PCR reactions. PCR products were run on 1.5\% agarose gel with 100 bp ladder (New England Biolabs, Cambridge, MA) and visualized on an iBright digital gel imager (Thermo Fisher, Waltham, MA).

Statistical analysis. For boxplots, the middle line represents median value, upper and lower box regions correspond to third and first quartiles (75th and 25th percentiles) and whiskers represent 1.5 times the interquartile range. All plots and analyses were generated in R v4.0. All raw data, analysis, and scripts for generation of figures are available on GitHub. Fiji/imageJ macros and CellProfiler pipelines are available on GitHub. Data were subjected to a Shapiro normality test before selecting the appropriate parametric or non-parametric statistical test. All experiments were repeated a minimum of three times except where noted, and confocal and electron microscopy images were chosen from at least five random fields examined.

Received: 1 December 2020; Accepted: 20 January 2021

Published online: 18 February 2021

\section{References}

1. Kalluri, R. \& LeBleu, V. S. The biology, function, and biomedical applications of exosomes. Science 367, 1-17 (2020).

2. Colombo, M., Raposo, G. \& Théry, C. Biogenesis, secretion, and intercellular interactions of exosomes and other extracellular vesicles. Ann. Rev. Cell. Dev. Biol. 30, 255-289 (2014).

3. Sabapatha, A., Gercel-taylor, C. \& Taylor, D. D. Specific isolation of placenta-derived exosomes from the circulation of pregnant women and their immunoregulatory consequences. Am. J. Reprod. Immunol. 56, 345-355 (2006).

4. Stefanski, A. L. et al. Murine trophoblast-derived and pregnancy-associated exosome-enriched extracellular vesicle microRNAs: Implications for placenta driven effects on maternal physiology. PLoS ONE https://doi.org/10.1371/journal.pone.0210675 (2019).

5. Salomon, C. et al. A gestational profile of placental exosomes in maternal plasma and their effects on endothelial cell migration. PLoS ONE https://doi.org/10.1371/journal.pone.0098667 (2014).

6. Nguyen, S. L. et al. Quantifying murine placental extracellular vesicles across gestation and in preterm birth data with tidyNano: A computational framework for analyzing and visualizing nanoparticle data in R. PLoS ONE https://doi.org/10.1371/journ al.pone.0218270 (2019).

7. Salomon, C. et al. Gestational diabetes mellitus is associated with changes in the concentration and bioactivity of placenta-derived exosomes in maternal circulation across gestation. Diabetes https://doi.org/10.2337/db15-0966 (2016).

8. Ospina-Prieto, S. et al. MicroRNA-141 is upregulated in preeclamptic placentae and regulates trophoblast invasion and intercellular communication. Transl. Res. 172, 61-72 (2016).

9. Tong, M., Chen, Q., James, J. L., Stone, P. R. \& Chamley, L. W. Micro- and nano-vesicles from first trimester human placentae carry Flt-1 and levels are increased in severe preeclampsia. Front. Endocrinol. (Lausanne). https://doi.org/10.3389/fendo.2017.00174 (2017).

10. Tannetta, D., Collett, G., Vatish, M., Redman, C. \& Sargent, I. Syncytiotrophoblast extracellular vesicles-Circulating biopsies reflecting placental health. Placenta 52, 134-138 (2017).

11. Atay, S., Gercel-Taylor, C. \& Taylor, D. D. Human trophoblast-derived exosomal fibronectin induces pro-inflammatory IL-1 $\beta$ production by macrophages. Am. J. Reprod. Immunol. 66, 259-269 (2011).

12. Mincheva-Nilsson, L. et al. Placenta-derived soluble MHC Class I chain-related molecules down-regulate NKG2D receptor on peripheral blood mononuclear cells during human pregnancy: A possible novel immune escape mechanism for fetal survival. $J$. Immunol. 176, 3585-3592 (2006).

13. Stenqvist, A.-C., Nagaeva, O., Baranov, V. \& Mincheva-Nilsson, L. Exosomes secreted by human placenta carry functional fas ligand and TRAIL molecules and convey apoptosis in activated immune cells, suggesting exosome-mediated immune privilege of the fetus. J. Immunol. 191, 5515-5523 (2013).

14. Kshirsagar, S. K. et al. Immunomodulatory molecules are released from the first trimester and term placenta via exosomes. Placenta 33, 982-990 (2012).

15. Delorme-Axford, E. et al. Human placental trophoblasts confer viral resistance to recipient cells. Proc. Natl. Acad. Sci. U. S. A. 110, 12048-12053 (2013).

16. Salomon, C. et al. Extravillous trophoblast cells-derived exosomes promote vascular smooth muscle cell migration. Front. Pharmacol. https://doi.org/10.3389/fphar.2014.00175 (2014).

17. Tong, M. et al. Placental nano-vesicles target to specific organs and modulate vascular tone in vivo. Hum. Reprod. 32, 2188-2198 (2017). 
18. Sheller-Miller, S., Choi, K., Choi, C. \& Menon, R. Cyclic-recombinase-reporter mouse model to determine exosome communication and function during pregnancy. Am. J. Obstet. Gynecol. 221(502), e1-502.e12 (2019).

19. Chakarov, S. et al. Two distinct interstitial macrophage populations coexist across tissues in specific subtissular niches. Science 363, 1-15 (2019).

20. Yu, Y. R. A. et al. A protocol for the comprehensive flow cytometric analysis of immune cells in normal and inflamed murine non-lymphoid tissues. PLoS ONE https://doi.org/10.1371/journal.pone.0150606 (2016).

21. Hoshino, A. et al. Tumour exosome integrins determine organotropic metastasis. Nature 527, 329-335 (2015).

22. Kapp, T. G. et al. A comprehensive evaluation of the activity and selectivity profile of ligands for RGD-binding integrins. Sci. Rep. https://doi.org/10.1038/srep39805 (2017).

23. Sroka, T. C., Pennington, M. E. \& Cress, A. E. Synthetic D-amino acid peptide inhibits tumor cell motility on laminin-5. Carcinogenesis 27, 1748-1757 (2006).

24. Susaki, E. A. et al. Whole-brain imaging with single-cell resolution using chemical cocktails and computational analysis. Cell 157, 726-739 (2014).

25. Muzumdar, M. D., Tasic, B., Miyamichi, K., Li, N. \& Luo, L. A global double-fluorescent cre reporter mouse. Genesis 45, 593-605 (2007).

26. Khosrotehrani, K., Johnson, K. L., Guégan, S., Stroh, H. \& Bianchi, D. W. Natural history of fetal cell microchimerism during and following murine pregnancy. J. Reprod. Immunol. 66, 1-12 (2005).

27. Fujiki, Y., Johnson, K. L., Tighiouart, H., Peter, I. \& Bianchi, D. W. Fetomaternal trafficking in the mouse increases as delivery approaches and is highest in the maternal lung. Biol. Reprod. 79, 841-848 (2008).

28. Pritchard, S., Wick, H. C., Slonim, D. K., Johnson, K. L. \& Bianchi, D. W. Comprehensive analysis of genes expressed by rare microchimeric fetal cells in the maternal mouse lung. Biol. Reprod. 87, 1-6 (2012).

29. Ridder, K. et al. Extracellular vesicle-mediated transfer of genetic information between the hematopoietic system and the brain in response to inflammation. PLoS Biol. https://doi.org/10.1371/journal.pbio.1001874 (2014).

30. Zomer, A. et al. In vivo imaging reveals extracellular vesicle-mediated phenocopying of metastatic behavior. Cell 161, 1046-1057 (2015).

31. Hann, S., Kvenvold, L., Newby, B. N., Hong, M. \& Warman, M. L. A Wisp3 Cre-knockin Allele produces efficient recombination in spermatocytes during early prophase of meiosis I. PLoS ONE https://doi.org/10.1371/journal.pone.0075116 (2013).

32. Southcombe, J., Tannetta, D., Redman, C. \& Sargent, I. The immunomodulatory role of syncytiotrophoblast microvesicles. PLoS ONE https://doi.org/10.1371/journal.pone.0020245 (2011).

33. Mor, G., Cardenas, I., Abrahams, V. \& Guller, S. Inflammation and pregnancy: The role of the immune system at the implantation site. Ann. N. Y. Acad. Sci. 1221, 80-87 (2011).

34. Bedoret, D. et al. Lung interstitial macrophages alter dendritic cell functions to prevent airway allergy in mice. J. Clin. Invest. 119, 3723-3738 (2009).

35. Grindheim, G., Estensen, M. E., Langesaeter, E., Rosseland, L. A. \& Toska, K. Changes in blood pressure during healthy pregnancy: A longitudinal cohort study. J. Hypertens. 30, 342-350 (2012).

36. Jamieson, D. J. et al. H1N1 2009 influenza virus infection during pregnancy in the USA. Lancet 374, 451-458 (2009).

37. Neuzil, K. M., Reed, G. W., Mitchel, E. F., Simonsen, L. \& Griffin, M. R. Impact of influenza on acute cardiopulmonary hospitalizations in pregnant women. Am. J. Epidemiol. 148, 1094-1102 (1998).

38. Esmonde, T. F., Herdman, G. \& Anderson, G. Chickenpox pneumonia: An association with pregnancy. Thorax 44, 812-815 (1989).

39. Lamont, R. F. et al. Varicella-zoster virus (chickenpox) infection in pregnancy. BJOG 118, 1155-1162 (2011).

40. Kim, S. et al. Effect of pregnancy in asthma on health care use and perinatal outcomes. J. Allergy Clin. Immunol. 136, 1215-1223 (2015).

41. Das, T. K., Moutquin, J. M. \& Parent, J. G. Effect of cigarette smoking on maternal airway function during pregnancy. Am. J. Obstet. Gynecol. 165, 675-679 (1991).

42. Erlebacher, A., Vencato, D., Price, K. A., Zhang, D. \& Glimcher, L. H. Constraints in antigen presentation severely restrict T cell recognition of the allogeneic fetus. J. Clin. Invest. 117, 1399-1411 (2007).

43. Holland, O. J. et al. Minor histocompatibility antigens are expressed in syncytiotrophoblast and trophoblast debris: Implications for maternal alloreactivity to the fetus. Am. J. Pathol. 180, 256-266 (2012).

44. Taglauer, E. S., Yankee, T. M. \& Petroff, M. G. Maternal PD-1 regulates accumulation of fetal antigen-specific CD8 ${ }^{+}$T cells in pregnancy. J. Reprod. Immunol. 80, 12-21 (2009).

45. Villarroya-Beltri, C., Baixauli, F., Gutiérrez-Vázquez, C., Sánchez-Madrid, F. \& Mittelbrunn, M. Sorting it out: Regulation of exosome loading. Sem. Cancer Biol. 28, 3-13 (2014).

46. Costa-Silva, B. et al. Pancreatic cancer exosomes initiate pre-metastatic niche formation in the liver. Nat. Cell Biol. 17, 816-826 (2015).

47. Yousif, L. F., Di Russo, J. \& Sorokin, L. Laminin isoforms in endothelial and perivascular basement membranes. Cell Adhes. Migr. 7, 101-110 (2013)

48. Costanzo, V., Bardelli, A., Siena, S. \& Abrignani, S. Exploring the links between cancer and placenta development. Open Biol. https ://doi.org/10.1098/rsob.180081 (2018).

49. Schmorl, G. Pathologisch-anatomische Untersuchunge uber Puerperal-Eklampsie. (Verlag, 1893).

50. Bianchi, D. W., Zickwolf, G. K., Weil, G. J., Sylvester, S. \& Demaria, M. A. Male fetal progenitor cells persist in maternal blood for as long as 27 years postpartum. Proc. Natl. Acad. Sci. U. S. A. 93, 705-708 (1996).

51. Evans, P. C. et al. Long-term fetal microchimerism in peripheral blood mononuclear cell subsets in healthy women and women with scleroderma. Blood 93, 2033-2037 (1999).

52. Wang, Y. et al. Fetal cells in mother rats contribute to the remodeling of liver and kidney after injury. Biochem. Biophys. Res. Commun. 325, 961-967 (2004).

53. Jasti, S., Farahbakhsh, M., Nguyen, S., Petroff, B. K. \& Petroff, M. G. Immune response to a model shared placenta/tumor-associated antigen reduces cancer risk in parous mice. Biol. Reprod. 96, 134-144 (2017).

54. van der Vlist, E. J., Nolte-'t Hoen, E. N. M., Stoorvogel, W., Arkesteijn, G. J. A. \& Wauben, M. H. M. Fluorescent labeling of nanosized vesicles released by cells and subsequent quantitative and qualitative analysis by high-resolution flow cytometry. Nat. Protoc. 7, 1311-1326 (2012)

55. Helft, J. et al. GM-CSF mouse bone marrow cultures comprise a heterogeneous population of CD11c+MHCII+ macrophages and dendritic cells. Immunity 42, 1197-1211 (2015).

56. Chen, H. et al. A bioconductor package for an integrated mass cytometry data analysis pipeline. PLoS Comput. Biol. https://doi. org/10.1371/journal.pcbi.1005112 (2016).

\section{Acknowledgements}

The authors thank lab members including Geoffrey Grzesiak, Sarika Kshirsagar, and Cole McCutcheon for technical assistance and useful discussions; Alicia Withrow for assistance with transmission electron microscopy; and Melinda Frame (MSU Center for Advanced Microscopy) for imaging assistance in confocal microscopy. The 
authors also thank Matthew Bernard (MSU Flow Cytometry Core Facility) with assistance in flow cytometry panels and procedures.

\title{
Author contributions
}

S.L.N. and M.G.P. conceived of the original ideas and experiments. S.L.N. carried out the experiments, analyzed and the data and presented it in visual format, and led in the writing of the manuscript. S.H.A. contributed to the ideas of experiments, assisted in carrying out experiments, and assisted in data visualization. J.W.G. assisted with development and validation of vesicle purification protocols. B.W.C. assisted with development and validation of placental explant cultures. D.W.A. analyzed histopathology of placental explants and assisted with interpretation of the results. R.A. assisted with 3 dimensional visualization, analysis, and interpretation of lung tissue images. M.G.P. acquired funding for the study, and supervised overall direction and planning of the research as well as the writing and editing of the manuscript. All authors provided input in the research design, analysis, and writing of the manuscript.

\section{Funding}

This research was supported by grants HD091429, (MGP) and MSU AgBioResearch/NIFA MICL02447 (MGP). SLN was supported by the Integrative Pharmacological Sciences Training Program T32GM092715.

\section{Competing interests}

The authors declare no competing interests.

\section{Additional information}

Supplementary Information The online version contains supplementary material available at https://doi. org/10.1038/s41598-021-82752-w.

Correspondence and requests for materials should be addressed to M.G.P.

Reprints and permissions information is available at www.nature.com/reprints.

Publisher's note Springer Nature remains neutral with regard to jurisdictional claims in published maps and institutional affiliations.

\begin{abstract}
Open Access This article is licensed under a Creative Commons Attribution 4.0 International License, which permits use, sharing, adaptation, distribution and reproduction in any medium or format, as long as you give appropriate credit to the original author(s) and the source, provide a link to the Creative Commons licence, and indicate if changes were made. The images or other third party material in this article are included in the article's Creative Commons licence, unless indicated otherwise in a credit line to the material. If material is not included in the article's Creative Commons licence and your intended use is not permitted by statutory regulation or exceeds the permitted use, you will need to obtain permission directly from the copyright holder. To view a copy of this licence, visit http://creativecommons.org/licenses/by/4.0/.
\end{abstract}

(C) The Author(s) 2021 